\title{
Spatialization of ECOS method at micro-basin level in rural development-oriented
}

\section{ecotourism planning}

Tuğba Kiper ${ }^{1}$, Osman Uzun*2, Oğuz Ateş ${ }^{3}$

${ }^{1}$ Tekirdag Namık Kemal University, Faculty of Fine Arts, Design and Architect, Tekirdag, Turkey.

${ }^{2}$ Düzce University, Faculty of Forestry, Düzce, Turkey.

${ }^{3}$ Kırklareli University, Faculty of Architect, Kırklareli, Turkey.

*Corresponding author:

E-mail: oguzates@klu.edu.tr, tel: +90 5443407940

\section{GRAPHICAL ABSTRACT}

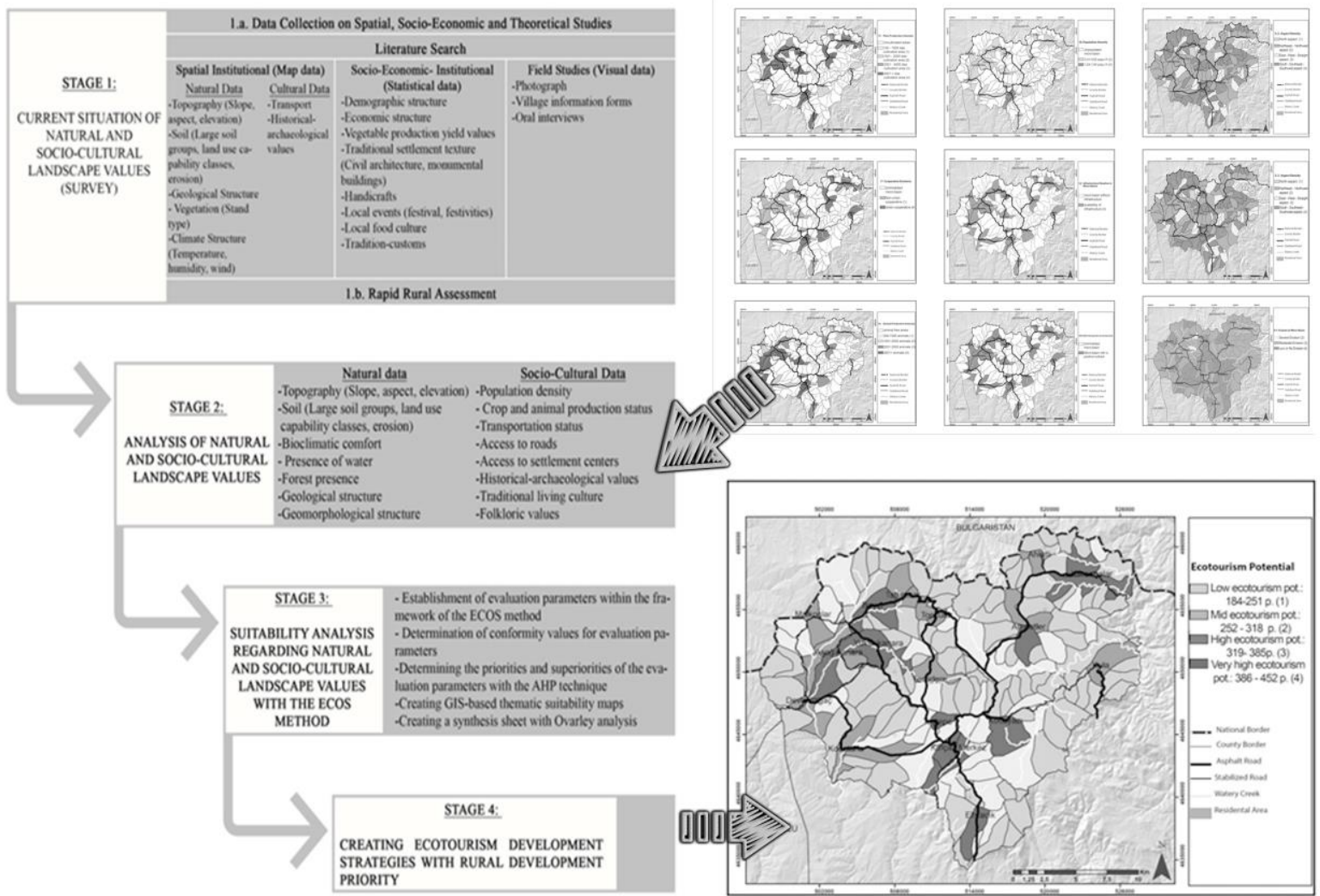




\section{ABSTRACT}

This research discusses the prediction that "within the framework of the principles of sustainable development; in regions deemed suitable for ecotourism activities, spatial planning is required, which includes a 'spatial sensitivity', 'participatory understanding' and 'holistic use'". Within the framework of the hypothesis developed; a new method approach was developed for Kofçaz/Kırklareli sample, located in northwest Turkey, that could be incorporated into future ecotourism policies and plans with ECOS and AHP Method, which can be adapted to the space on a micro-basin basis.

During the inventory phase of the study and determination of the current situation, a database was created in the GIS environment on the basis of 145 micro-basins determined by the philosophy of the basin and sub-basin of the data on natural and cultural landscape elements. 6 basic criteria, 23 evaluation factors and 73 sub-evaluation criteria were determined with ECOS method developed in the light of RRA Technique and literature findings to be applied in the field. To determine the priority values and ranking of the main and lower criteria, weight scores related to evaluation criteria were determined from 12 individuals via the AHP technique. Conformity values and conformity coefficients for the specified basic and sub-criteria were analyzed at 145 micro basin levels and thematic maps were created. With overlay analysis, synthesis pad was created, appropriate areas for ecotourism were determined, and ecotourism management strategies focused on rural development were developed.

These results can be integrated into basin-scale spatial planning that stands out both nationally and internationally.

Keywords: Ecoturism, ECOS, Kırklareli. 


\section{Introduction}

Rural areas are complex structures with limited relations with urban centers, that show diverse income distribution, limited access to services, basic features such as history, natural, local values, climate, as well as the development of settlement models linked to areas connected to natural structure and where cultural interaction occurs (Ortiz-Guerrero, 2013). At this point; supranational institutions such as the World Bank (WB), the United Nations (UN), the Organization for Economic Development and Cooperation (OECD) and the European Union (EU) have developed approaches to rural development. The World Bank (WB) promotes sustainable and attractiveness of rural areas, harmonization of economic, social, cultural, environmental and technological changes, poverty reduction within the framework of the United Nations Millennium Development Goals; OECD aims at developing economic growth linked to agricultural production, as well as the development of competitiveness based on various sectors such as tourism, manufacturing, information and infrastructure; on the other hand, the European Union promotes quality of life and diversification of the rural economy in rural areas (Gülçubuk et al., 2016; Çelik, 2006; Yenigül, 2017).

Likewise, with the change in their understanding of rural development; tourism has been a solution in the focus of development, and especially it is aimed to improve ecotourism as a priority. As a matter of fact, in the report titled "Strategy for Rural Europe" prepared by ECOVAST; tourism is envisaged in the revival of rural economies (ÇEKÜL, 2012). By the United Nations; 2002 was declared as "International Year of Ecotourism," and 2017 was declared as "Sustainable Tourism Year for Development". So much so that ecotourism contributes to the economic improvement of the local community with a focus on protecting natural resources, local identity and cultural values (Ashok et al., 2017; Kiper, 2012; Gigović et al., 2016; TIES, 2015). However, as Roger and Bhatta (2013) indicated; "In the effectiveness of ecotourism in sustainable development, it is important how it is planned, implemented and managed". Sustainable development is based on a viable economy, responsible governance, social cohesion and ecological integrity (Alexander and Whitehouse, 2004; Cheia, 2013). Ecotourism has also been cited by many researchers as a tool for achieving sustainable 
development (Li, 2004; Telfer and Sharpley, 2008; Tran and Walter in 2013; Moeljad, 2015; Safarabadi, 2016; Seifi and Janbaz Ghobad, 2017).

In the study; on the basis of the development of a new methodology that can be included in ecotourism plans in rural areas; answers to the questions such as "What should tourism development be spatially like in areas preceded as ecotourism development areas in high scale plan decisions?" and "What should be the prediction of development-first ecotourism strategies?" were searched. In this direction, in the case of the Forties/Kofçaz micro basins in northwestern Turkey, it is aimed to plan a sustainable, economically viable and socially acceptable ecotourism based on development-first local identity values and to develop management strategies.

\section{Materials and methods}

\subsection{Materials}

In the selection of the main material of the study, sub-basins and micro-basins within each sub-basin were taken into account, taking into account natural thresholds rather than administrative boundaries. However, in many ecology-based spatial planning studies, it is stated that the basin and micro basin scale should be used effectively for rural and urban areas (Forman, 1995; Jones et al., 1997; Uzun et al., 2015; Kiper et al., 2017; Yüksel et al., 2020). In this context; the study was based on the Kofçaz/Kırklareli micro-basins and on 145 micro basins in the processing, evaluation and analysis of data within the framework of the method process. While creating micro-basins; based on the subbasins used by DSI; micro-basins within sub-basins were determined based on water separation lines and leveling curved maps (Table 1).

Table 1. Coding studies of Meriç and Marmara main basin micro-basins

\begin{tabular}{|c|c|c|c|c|c|}
\hline $\begin{array}{c}\text { Turkey } \\
\text { main } \\
\text { watershed }\end{array}$ & $\begin{array}{c}\text { Turkey main } \\
\text { watershed } \\
\text { code }\end{array}$ & $\begin{array}{c}\text { Subwater } \\
\text { no }\end{array}$ & $\begin{array}{c}\text { Subwater } \\
\text { code }\end{array}$ & Micro-basins code & $\begin{array}{c}\text { Micro-basins } \\
\text { count }\end{array}$ \\
\hline Meriç & 01 & 30 & 130 & $130001-130086$ & 86 \\
\hline Marmara & 02 & 50 & 250 & $225001-225059$ & 59 \\
\hline \multicolumn{7}{|r|}{} & Total & 145 \\
\hline
\end{tabular}


The working field of the study is located in the Thrace part of the Marmara Region at the foot of the Yildiz (Istranca) Mountains in northwestern Turkey. Kofçaz District was defined as the "rural center" and considered as the area where agro-ecotourism would be developed and was included in the "ecoagro tourism corridor" (Anonymous, 2009; Anonymous, 2014).

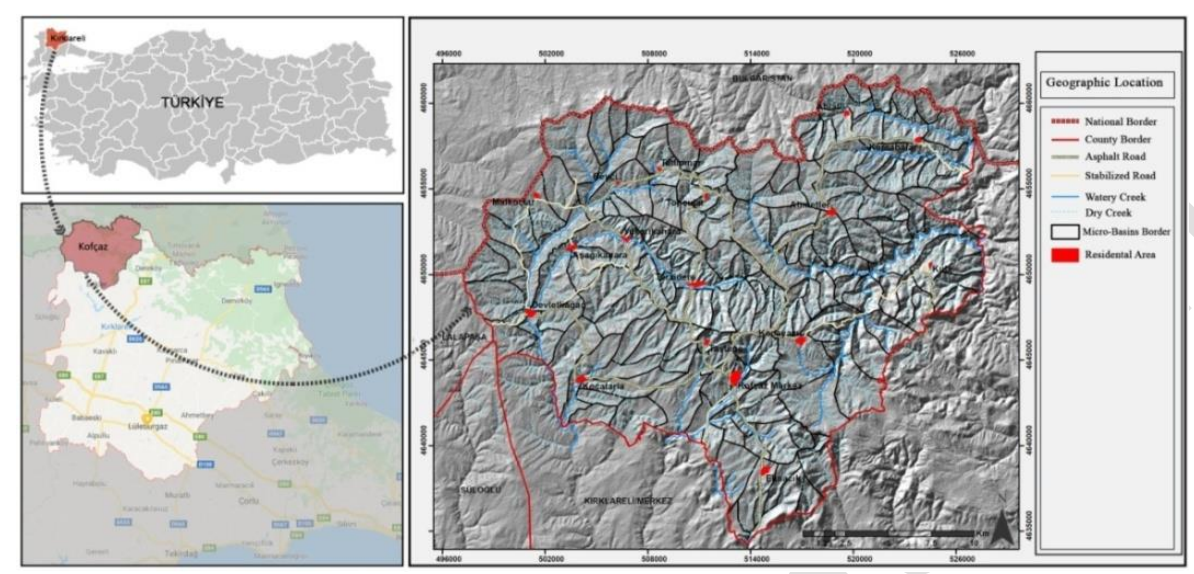

Figure 1. Location of study area

\subsection{Methods}

When the studies on the planning of ecotourism areas are analyzed, it is seen that methods like, Recreation Opportunities Spectrum (ROS), Water Recreation Opportunity Spectrum (WROS), Water and Land Recreation Opportunity Spectrum (WALROS), Tourism Sectorization Opportunity Spectrum (TSOS) and Forest Recreation Opportunity Spectrum (FROS) have been used. In this study, ECOS method created by combining and adapting Recreation Opportunities Spectrum (ROS) and Tourism Opportunities Spectrum (TOS) approaches was used. ECOS method in the study includes a technically usable, environmentally sustainable, socially acceptable, economically viable approach that supports local resource values (spatial sensitivity), takes into account the opinions and expectations of relevant stakeholders (participatory approach), where land and office work is carried out together, information-based, natural and cultural data are processed, stored and queried through GIS (based on the data information system). ECOS Method was used in the conformity analysis phase by detailing the evaluation criteria created by making various changes and adaptations in the field of 
study in Arc GIS environment at the lower-basin and micro-basin level. The general method was carried out in 4 basic stages (Figure 2).

\begin{tabular}{|c|c|c|c|c|}
\hline \multirow{13}{*}{$\begin{array}{l}\text { CURRENT SITUATION OF } \\
\text { NATURAL AND } \\
\text { SOCIO-CULTURAL } \\
\text { LANDSCAPE VALUES } \\
\text { (SURVEY) }\end{array}$} & \multicolumn{4}{|c|}{ 1.a. Data Collection on Spatial, Socio-Economic and Theoretical Studies } \\
\hline & \multicolumn{4}{|c|}{ Literature Search } \\
\hline & \multicolumn{2}{|c|}{ Spatial Institutional (Map data) } & \multirow{10}{*}{$\begin{array}{l}\text { Socio-Economic- Institutional } \\
\text { (Statistical data) } \\
\text {-Demographic structure } \\
\text {-Economic structure } \\
\text {-Vegetable production yield values } \\
\text {-Traditional settlement texture } \\
\text { (Civil architecture, monumental } \\
\text { buildings) } \\
\text {-Handicrafts } \\
\text {-Local events (festival, festivities) } \\
\text {-Local food culture } \\
\text {-Tradition-customs }\end{array}$} & \multirow{10}{*}{$\begin{array}{l}\text { Field Studies (Visual data) } \\
\text {-Photograph } \\
\text {-Village information forms } \\
\text {-Oral interviews }\end{array}$} \\
\hline & Natural Data & Cultural Data & & \\
\hline & -Topography (Slope, & -Transport & & \\
\hline & -Soil (Large soil & archacological & & \\
\hline & groups, land use ca- & values & & \\
\hline & & & & \\
\hline & -Geological Structure & & & \\
\hline & $\begin{array}{l}\text { - Vegetation (Stand } \\
\text { type) }\end{array}$ & & & \\
\hline & -Climate Structure & & & \\
\hline & $\begin{array}{l}\text { (Temperature, } \\
\text { humidity, wind) }\end{array}$ & & & \\
\hline & \multicolumn{4}{|c|}{ 1.b. Rapid Rural Assessment } \\
\hline
\end{tabular}

\begin{tabular}{|c|c|c|c|}
\hline $\begin{array}{l}\text { ANALYSIS OF NATURAL } \\
\text { AND SOCIO-CULTURAL } \\
\text { LANDSCAPE VALUES }\end{array}$ & \multicolumn{2}{|c|}{$\begin{array}{l}\text {-Topography (Slope, aspect, elevation) } \\
\text {-Soil (Large soil groups, land use } \\
\text { capability classes, erosion) } \\
\text {-Bioclimatic comfort } \\
\text { - Presence of water } \\
\text {-Forest presence } \\
\text {-Geological structure } \\
\text {-Geomorphological structure }\end{array}$} & $\begin{array}{l}\text { Socio-Cultural Data } \\
\text {-Population density } \\
\text { - Crop and animal production status } \\
\text {-Transportation status } \\
\text {-Access to roads } \\
\text {-Access to settlement centers } \\
\text {-Historical-archaeological values } \\
\text {-Traditional living culture } \\
\text {-Folkloric values }\end{array}$ \\
\hline $\begin{array}{l}\text { SUITAB } \\
\text { REGAR } \\
\text { AND SO } \\
\text { LANDS } \\
\text { WIT }\end{array}$ & $\begin{array}{l}\text { STAGE 3: } \\
\text { 3ILITY ANALYSIS } \\
\text { DDING NATURAL } \\
\text { OCIO-CULTURAL } \\
\text { SCAPE VALUES } \\
\text { TH THE ECOS } \\
\text { METHOD }\end{array}$ & $\begin{array}{l}\text { - Establishment } \\
\text { mework of the E } \\
\text { - Determination } \\
\text { rameters } \\
\text {-Determining th } \\
\text { luation parametc } \\
\text {-Creating GIS-b } \\
\text {-Creating a synt }\end{array}$ & $\begin{array}{l}\text { of evaluation parameters within the fra- } \\
\text { ECOS method } \\
\text { of conformity values for evaluation pa- } \\
\text { le priorities and superiorities of the eva- } \\
\text { ers with the AHP technique } \\
\text { ased thematic suitability maps } \\
\text { thesis sheet with Ovarley analysis }\end{array}$ \\
\hline & $\begin{array}{l}\text { CREAT } \\
\text { STRATI } \\
\text { PRIORI }\end{array}$ & $\begin{array}{l}\text { STAG } \\
\text { JG ECOTOUR } \\
\text { GIES WITH RL } \\
\text { Y }\end{array}$ & $\begin{array}{l}\text { GE 4: } \\
\text { ISM DEVELOPMENT } \\
\text { URAL DEVELOPMENT }\end{array}$ \\
\hline
\end{tabular}

Figure 2. Flowchart of the study method

Stage I: This stage is at the core of the approach that "supports local resource values". This section is supported in 2 subdivisions (Table 2).

Stage II: Developed based on office and field studies, this stage was conducted based on thematic maps based on GIS (Table 3). 
Table 2. 2-step method that supports local resource values

\begin{tabular}{|c|c|}
\hline 1. Chapter & 2. Chapter \\
\hline $\begin{array}{l}\text { Current due diligence based on spatial, socio- } \\
\text { economic criteria and field trips }\end{array}$ & Rapid Rural Assessment \\
\hline $\begin{array}{l}\text { - Subject (Rural development, rural planning, } \\
\text { ecotourism development, rural landscape } \\
\text { planning, ecotourism planning, ECOS method, } \\
\text { etc.) and literature (thesis, article, report) } \\
\text { studies on the field were examined. } \\
\text { - Prepared by the relevant institutions and } \\
\text { organizations; spatial and socio-economic } \\
\text { plans, reports, printed and/or numerical maps, } \\
\text { statistical data were provided at the upper scale. } \\
\text { - Village information forms and Rapid Rural } \\
\text { Assessment forms were prepared. } \\
\text { - Visual materials were provided with land works } \\
\text { and interviews were held with local people and } \\
\text { local authorities. }\end{array}$ & $\begin{array}{l}\text { This section constitutes the essence of the } \\
\text { participatory approach that "takes into account } \\
\text { the opinions and expectations of the relevant } \\
\text { stakeholders" within the framework of the } \\
\text { method. It is the stage at which the current } \\
\text { situation is determined and the answer to the } \\
\text { question of "Where" is determined. } \\
\text { RRA was carried out with } 12 \text { people consisting of } \\
\text { local institution representatives. }\end{array}$ \\
\hline
\end{tabular}

Table 3. Processes carried out within the scope of the current situation analysis and related data sources

\begin{tabular}{|c|c|c|}
\hline \multirow{11}{*}{ 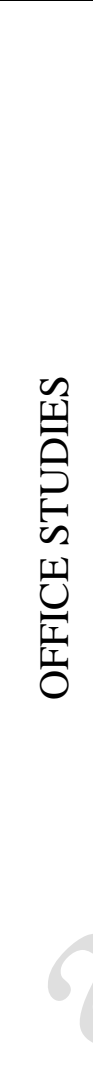 } & \multicolumn{2}{|l|}{ Natural Data } \\
\hline & Source & The data obtained \\
\hline & $\begin{array}{l}\text { General Command of Mapping 1/25.000 } \\
\text { scale digital map }\end{array}$ & Elevation groups (m), Slope (\%), \\
\hline & $\begin{array}{l}\text { General Directorate of Rural Services } \\
1 / 25.000 \text { scale digital soil map }\end{array}$ & $\begin{array}{l}\text { Large soil groups, landuse capability classes, } \\
\text { erosion }\end{array}$ \\
\hline & $\begin{array}{l}\text { Climate data of the General Directorate } \\
\text { of Meteorology }\end{array}$ & Bioclimatic comfort \\
\hline & $\begin{array}{l}\text { Ministry of Agriculture and Forestry } \\
\text { Kirklareli Forestry Directorate Forest } \\
\text { Management Plan }\end{array}$ & Forest stand canopy cover \\
\hline & \multicolumn{2}{|l|}{ Culturel Data } \\
\hline & Population data & Population distribution, population density \\
\hline & $\begin{array}{l}\text { Kirklareli Provincial General Directorate } \\
\text { of Agriculture and Forestry } \\
\text { Kofçaz District Directorate of } \\
\text { Agriculture and Forestry }\end{array}$ & Agricultural and animal production \\
\hline & $\begin{array}{l}\text { General Command of Mapping 1/25.000 } \\
\text { scale digital map }\end{array}$ & $\begin{array}{l}\text { Transportation status, access to road, access to } \\
\text { residential areas }\end{array}$ \\
\hline & $\begin{array}{llll}\text { Kirklareli } & \text { Culture } & \text { and } & \text { Tourism } \\
\text { Directorate } & & & \end{array}$ & $\begin{array}{l}\text { Historical and archeological structures, tourist } \\
\text { infrastructure facilities, socio-cultural values, } \\
\text { local architecture }\end{array}$ \\
\hline & \multicolumn{2}{|c|}{$\begin{array}{l}\text { Data Usage Type: Field observations, interviews with local people, interviews with central } \\
\text { and local government representatives }\end{array}$} \\
\hline 至 & \multicolumn{2}{|c|}{ Hydrology, landforms, vegetation } \\
\hline 崖它 & \multicolumn{2}{|c|}{$\begin{array}{l}\text { Historical background of the village (foundation year, ethnicity, etc.), livelihoods, } \\
\text { infrastructure situation }\end{array}$} \\
\hline & \multicolumn{2}{|c|}{ Public perspective on ecotourism } \\
\hline
\end{tabular}


Stage III: ECOS method was used to demonstrate the potential of ecotourism and to determine the priority areas for ecotourism. ECOS method is an important tool in determining resource values and ecotourism opportunities for ecotourism and determining relative priorities for ecotourism activities and contributes to ecotourism planning and management (Boyd and Butler, 1996; Fagence, 2001; Neth, 2008; Ajlaoni, 2011). According to With (2011), ECOS is a tool for the analysis of ecotourismprior development and is crucial for developing environmentally and socio-economically sustainable ecotourism strategies (Poyyamoli, 2018). The ECOS method was first used by Boyd and Butler in 1996. Within the framework of the ECOS method, 8 basic criteria ((1) accessibility, (2) the relationship of ecotourism with other sources, (3) regional attractiveness, (4) touristic infrastructure, (5) the ability and knowledge of ecotourists, (6) the level of social interaction, (7) the impact of visitors on the source of ecotourism and the management style developed for the long-term protection of the area) used by Boyd and Butler (1996) were then developed by applying various changes and adaptations, and these criteria were evaluated in various forms (Table 4).

Table 4. Evaluation criteria based on the ECOS method

\begin{tabular}{|c|c|c|c|c|c|c|c|c|c|c|c|c|c|c|c|}
\hline Evaluation criteria & 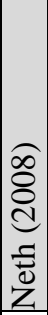 & 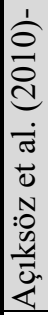 & 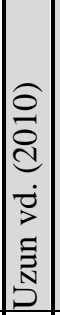 & 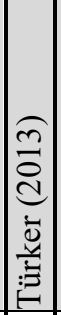 & 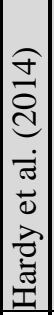 & 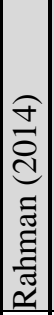 & 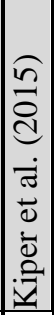 & 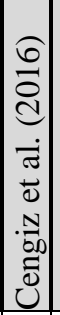 & 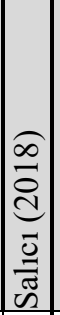 & 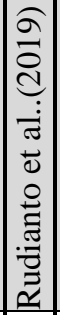 & 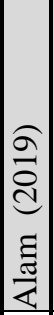 & 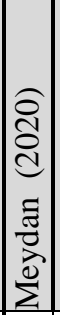 & 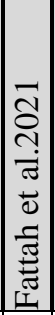 & 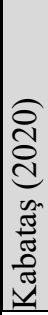 & 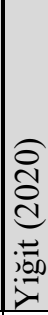 \\
\hline Accessibility & $\mathrm{x}$ & & & $\mathrm{x}$ & $\mathrm{x}$ & $\mathrm{x}$ & $\mathrm{x}$ & & $\mathrm{x}$ & $\mathrm{x}$ & $\mathrm{x}$ & & $\mathrm{x}$ & $\mathrm{x}$ & $\mathrm{x}$ \\
\hline Level of responding to distinct ecotourism activities & $\mathrm{x}$ & & & & $\mathrm{X}$ & $\mathrm{x}$ & & & $\mathrm{x}$ & $\mathrm{x}$ & & & & & \\
\hline Attractions in a region & $\mathrm{x}$ & & & & $\mathrm{x}$ & $\mathrm{x}$ & & & & $\mathrm{x}$ & $\mathrm{x}$ & & & & \\
\hline Available tourism infrastructure & $\mathrm{x}$ & & & $\mathrm{x}$ & & & $\mathrm{x}$ & & $\mathrm{x}$ & $\mathrm{x}$ & $\mathrm{x}$ & & $\mathrm{x}$ & $\mathrm{x}$ & $\mathrm{x}$ \\
\hline Level of user skill and knowledge required & $\mathrm{x}$ & & & & $\mathrm{x}$ & & & & $\mathrm{x}$ & & $\mathrm{x}$ & & $\mathrm{x}$ & & \\
\hline The correlation between ecotourism and other sources & & & & & $\mathrm{x}$ & $\mathrm{x}$ & $\mathrm{x}$ & & $\mathrm{x}$ & $\mathrm{x}$ & $\mathrm{x}$ & & $\mathrm{x}$ & & \\
\hline Effects of visitors to ecotourism source & & & & & & & & & & & & & $\mathrm{x}$ & & \\
\hline $\begin{array}{l}\text { Type of management needed to ensure the viability of areas } \\
\text { on along-term basis }\end{array}$ & $\mathrm{x}$ & & & $\mathrm{x}$ & & & & & & $\mathrm{x}$ & & & & & \\
\hline Source diversity of the focus & & & & & & & & & $\mathrm{x}$ & & & & & & $\mathrm{x}$ \\
\hline Available tourism superstructure & & & & $\mathrm{x}$ & & & $\mathrm{x}$ & & $\mathrm{x}$ & & & & & $\mathrm{X}$ & $\mathrm{X}$ \\
\hline Sociocultural structure & & $\mathrm{x}$ & $\mathrm{x}$ & $\mathrm{x}$ & & & $\mathrm{x}$ & $\mathrm{x}$ & & & & & & $\mathrm{x}$ & $\mathrm{X}$ \\
\hline Economic structure & & $\mathrm{X}$ & $\mathrm{x}$ & $\mathrm{x}$ & & & & $\mathrm{x}$ & & & & & & $\mathrm{x}$ & $\mathrm{x}$ \\
\hline Bio-physical structure & & $\mathrm{X}$ & $\mathrm{x}$ & & & & & $\mathrm{x}$ & & & $\mathrm{x}$ & & & & \\
\hline Source diversity of the naturel & & & & & & & $\mathrm{x}$ & & $\mathrm{x}$ & & & & & $\mathrm{x}$ & \\
\hline Visual attractiveness & & & & & & & & & $\mathrm{x}$ & & & & & & \\
\hline Protected ecosystem elements & & & & & & & & & $\mathrm{x}$ & & & & & & \\
\hline
\end{tabular}


Making use of 18 different studies regarding the EKOS method used in the study (Boyd and Butler, 1996; Topay, 2003; Bi, 2005, Khalid et al. 2010; Khalid, Nasır and Ahmad, 2010; Jurowski, 2010; Aç1ksöz et al. 2010; Uzun et al. 2010; Türker, 2013; Yassera and Sharma, 2014; Gültekin, 2014; Uzun et al., 2015; Kiper et al., 2015; Salıc1 2018; Kabataş, 2020;), 6 basic criteria, 23 evaluation factors and 73 sub-evaluation criteria were determined in the field of research (Table 5).

The high number of criteria and basic criteria evaluated and the fact that they are varied has increased the sensitivity of the ecotourism study. The 4-point Likert scale (4: Very high, 3: High, 2: Medium, 1: Low) was used for the numerical expression of the conformity values of the criteria and subcriteria. Studies by Türker (2013) and Görmüş (2017) were referred in the scoring.

Table 5. Evaluation criteria and sub-units of the ECOS method

\begin{tabular}{|c|c|c|c|c|}
\hline \multicolumn{4}{|c|}{ ECOS EVALUATION CRITERIA AND SUB-UNITS } & $\begin{array}{c}\text { SCORE* } \\
(1-4)\end{array}$ \\
\hline \multicolumn{5}{|c|}{ 1- Access to roads and volume situation } \\
\hline \multirow{14}{*}{ 1. ACCESSIBILITY } & \multirow{2}{*}{\multicolumn{2}{|c|}{$\begin{array}{l}1.11-\text { Access to } \\
\text { roads }\end{array}$}} & $\begin{array}{l}\text { Micro-basin where } \\
\text { the mainland passes }\end{array}$ & 4 \\
\hline & & & $\begin{array}{l}\text { Micro-basin where } \\
\text { stablized roads }\end{array}$ & 2 \\
\hline & \multirow{4}{*}{$\begin{array}{l}1.2 \text { Access } \\
\text { density to roads }\end{array}$} & \multirow{4}{*}{$\begin{array}{l}\text { Road rate } \\
\text { (length) } \\
\text { per } \mathrm{km} 2 \\
\text { in micro- } \\
\text { basin }\end{array}$} & $0-400 \mathrm{~m} / \mathrm{km} 2$ & 1 \\
\hline & & & $400-800 \mathrm{~m} / \mathrm{km} 2$ & 2 \\
\hline & & & $800-1200 \mathrm{~m} / \mathrm{km} 2$ & 3 \\
\hline & & & $1200 \mathrm{~m}>\mathrm{km} 2$ & 4 \\
\hline & & \multicolumn{3}{|c|}{ 2- Access to Settlements } \\
\hline & \multirow{4}{*}{\multicolumn{2}{|c|}{$\begin{array}{l}2.1 \text { Access from micro- } \\
\text { basin to settlements }\end{array}$}} & $\begin{array}{l}\text { Tertiary micro-basin } \\
\text { in contact with } \\
\text { settlement }\end{array}$ & 4 \\
\hline & & & $\begin{array}{l}\text { Secondary micro- } \\
\text { basin in contact with } \\
\text { settlement }\end{array}$ & 3 \\
\hline & & & $\begin{array}{l}\text { Primary micro-basin } \\
\text { in contact with } \\
\text { settlement }\end{array}$ & 2 \\
\hline & & & $\begin{array}{l}\text { Quaternary micro- } \\
\text { basin in contact with } \\
\text { settlement }\end{array}$ & 1 \\
\hline & & \multicolumn{3}{|c|}{ 3- Access to Ecotourism Resources } \\
\hline & & & $0-5000 \mathrm{~m}$ & 4 \\
\hline & & & $5001-10000 \mathrm{~m}$ & 3 \\
\hline
\end{tabular}




\begin{tabular}{|c|c|c|c|c|}
\hline & \multirow{2}{*}{\multicolumn{2}{|c|}{$\begin{array}{l}3.1 \text { Access from micro- } \\
\text { basin to recreational activity } \\
(\mathrm{m})\end{array}$}} & $10001-15.000 \mathrm{~m}$ & 2 \\
\hline & & & $15.001 \mathrm{~m}>$ & 1 \\
\hline & \multirow{4}{*}{\multicolumn{2}{|c|}{$\begin{array}{l}3.2 \text { Access from micro- } \\
\text { basin to water resources } \\
\text { (m) }\end{array}$}} & $\begin{array}{l}0-1000 \mathrm{~m} \text { access to } \\
\text { watery streams }\end{array}$ & 4 \\
\hline & & & $\begin{array}{l}1000-2000 \mathrm{~m} \text { access } \\
\text { to watery streams }\end{array}$ & 3 \\
\hline & & & $\begin{array}{l}2000-5000 \mathrm{~m} \text { access } \\
\text { to watery streams }\end{array}$ & 2 \\
\hline & & & $\begin{array}{l}5000>\mathrm{m} \text { access to } \\
\text { watery streams }\end{array}$ & 1 \\
\hline \multirow{28}{*}{$\begin{array}{l}\text { 2- LANDSCAPE } \\
\text { NATURAL } \\
\text { ATTRACTION } \\
\text { VALUES }\end{array}$} & \multicolumn{4}{|c|}{\begin{tabular}{|l|l|} 
& 4- Bioclimatic Comfort \\
\end{tabular}} \\
\hline & \multirow{2}{*}{\multicolumn{3}{|c|}{$\begin{array}{l}\text { 4.1 High bioclimatic comfort value }(18-230 \mathrm{C}) \\
\text { 4.2 Moderate bioclimatic comfort value }(13-18 \\
\text { 0C) }\end{array}$}} & 4 \\
\hline & & & & \\
\hline & & \multicolumn{3}{|c|}{ 5- Variety of Topographic Structures } \\
\hline & \multirow{4}{*}{$\begin{array}{l}5.1 \\
\text { Height Group } \\
\text { Variety }\end{array}$} & \multicolumn{2}{|c|}{$\begin{array}{l}\text { Micro-basin with } 4 \text { diff. height } \\
\text { groups }\end{array}$} & 4 \\
\hline & & \multicolumn{2}{|c|}{$\begin{array}{l}\text { Micro-basin with } 3 \text { diff. height } \\
\text { groups }\end{array}$} & 3 \\
\hline & & \multicolumn{2}{|c|}{$\begin{array}{l}\text { Micro-basin with } 2 \text { diff. height } \\
\text { groups }\end{array}$} & 2 \\
\hline & & \multicolumn{2}{|c|}{$\begin{array}{l}\text { Micro-basins with single group } \\
\text { height }\end{array}$} & 1 \\
\hline & \multirow{3}{*}{$\begin{array}{l}5.2 \text { Average } \\
\text { slope } \\
(\%)\end{array}$} & \multicolumn{2}{|c|}{$\begin{array}{l}\text { Micro-basin where the average } \\
\text { slope is } \% 0-18\end{array}$} & 4 \\
\hline & & \multicolumn{2}{|c|}{$\begin{array}{l}\text { Micro-basin where the average } \\
\text { slope is } \% 18-30\end{array}$} & 3 \\
\hline & & \multicolumn{2}{|c|}{$\begin{array}{l}\text { Micro-basin where the average } \\
\text { slope is } \% 30>\end{array}$} & 2 \\
\hline & \multirow{4}{*}{$\begin{array}{l}5.3 \\
\text { Aspect Density }\end{array}$} & \multicolumn{2}{|c|}{$\begin{array}{lll}\text { South, } & \text { southeast, } & \text { southwest } \\
\text { aspect }\end{array}$} & 4 \\
\hline & & \multicolumn{2}{|c|}{ East, west, straight aspect } & 3 \\
\hline & & \multicolumn{2}{|c|}{ Northeast, northeast aspect } & 2 \\
\hline & & \multicolumn{2}{|c|}{ North aspect } & 1 \\
\hline & 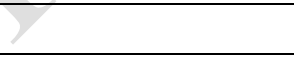 & \multicolumn{3}{|c|}{ 6- Forest Presence } \\
\hline & \multirow[t]{4}{*}{$\begin{array}{l}6.1 \text { Forest stand } \\
\text { canopy cover }\end{array}$} & \multicolumn{2}{|c|}{$\begin{array}{l}\% 10>\text { closed microbasin with } \\
\text { space }\end{array}$} & 4 \\
\hline & & \multicolumn{2}{|c|}{$\begin{array}{l}\% \quad 11-40 \text { loose } \quad \text { covered } \\
\text { microbasin }\end{array}$} & 3 \\
\hline & & \multicolumn{2}{|c|}{$\% 41-70$ mid covered microbasin } & 2 \\
\hline & & \multicolumn{2}{|c|}{$\% 71-100$ covered microbasin } & 1 \\
\hline & & \multicolumn{3}{|c|}{ 7- Water Presence } \\
\hline & \multirow{5}{*}{$\begin{array}{l}7.1 \text { Stream } \\
\text { density in } \\
\text { forest areas in } \\
\text { microbasins }\end{array}$} & \multicolumn{2}{|c|}{$\% 75-100$ stream density } & 4 \\
\hline & & \multicolumn{2}{|c|}{$\% 50-75$ stream density } & 3 \\
\hline & & $\% 25-50 \mathrm{st}$ & eam density & 2 \\
\hline & & $\% 0-25 \mathrm{str}$ & am density & 1 \\
\hline & & 8-Soil & & \\
\hline & 8.1 & Low or no & erosion & 4 \\
\hline & & Moderate & rosion & 3 \\
\hline
\end{tabular}




\begin{tabular}{|c|c|c|c|}
\hline & \multirow{2}{*}{$\begin{array}{l}\text { Erosion in } \\
\text { microbasin }\end{array}$} & Severe erosion & 2 \\
\hline & & Very severe erosion & 1 \\
\hline & \multirow{4}{*}{$\begin{array}{l}8.2 \text { Landuse } \\
\text { capability } \\
\text { classes in } \\
\text { microbasins }\end{array}$} & I., II , VII. Class LLC. & 4 \\
\hline & & III, VI. class LLLC & 3 \\
\hline & & IV. and V. class LLC & 2 \\
\hline & & VIII. classs LLC & 1 \\
\hline \multirow{14}{*}{$\begin{array}{l}\text { 3- LANDSCAPE } \\
\text { CULTURAL } \\
\text { ATTRACTION } \\
\text { VALUES }\end{array}$} & & \multicolumn{2}{|l|}{$\begin{array}{l}\text { 9- Folkloric Values (Traditional food, } \\
\text { handicrafts and festival, celebration, } \\
\text { ceremony etc.) }\end{array}$} \\
\hline & \multirow{3}{*}{$\begin{array}{l}9.1 \\
\text { Availability of } \\
\text { folkloric } \\
\text { values in } \\
\text { micro-basins }\end{array}$} & $\begin{array}{l}\text { Micro-basin with } 3 \text { folkloric } \\
\text { value }\end{array}$ & 4 \\
\hline & & $\begin{array}{l}\text { Micro-basin with } 2 \text { folkloric } \\
\text { value }\end{array}$ & \\
\hline & & $\begin{array}{l}\text { Micro-basin with } 1 \text { folkloric } \\
\text { value }\end{array}$ & 2 \\
\hline & & \multicolumn{2}{|c|}{ 10- Historical Archaeological Values } \\
\hline & \multirow{5}{*}{$\begin{array}{l}10.1 \\
\text { Accessibility } \\
\text { to historical } \\
\text { archaeological } \\
\text { values in } \\
\text { micro-basins }\end{array}$} & $\begin{array}{l}4 \quad \text { and more historical } \\
\text { archaeological values }\end{array}$ & 4 \\
\hline & & 3 historical archaeological values & 3 \\
\hline & & 2 historical archaeological values & 2 \\
\hline & & 1 historical archaeological values & 1 \\
\hline & & \multicolumn{2}{|l|}{ 11- Local Architectural Structures } \\
\hline & \multirow{2}{*}{$\begin{array}{l}11.1 . \\
\text { Micro-basins } \\
\text { with local } \\
\text { architectural } \\
\text { structures }\end{array}$} & 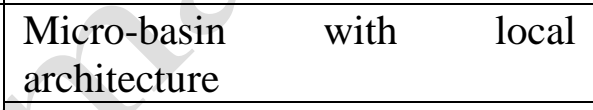 & 4 \\
\hline & & $\begin{array}{ccc}\text { Microbasin } & \text { without } & \text { local } \\
\text { architecture } & & \end{array}$ & 2 \\
\hline & & \multicolumn{2}{|l|}{ 12. Traditional Life Culture } \\
\hline & $\begin{array}{l}\text { 12.1 Diversity } \\
\text { of traditional } \\
\text { life culture in } \\
\text { micro-basins }\end{array}$ & $\begin{array}{l}\text { Micro-basin with a traditional } \\
\text { life culture }\end{array}$ & 4 \\
\hline \multirow{10}{*}{$\begin{array}{l}\text { 4- ECONOMIC } \\
\text { STRUCTURE } \\
\text { DIVERSITY }\end{array}$} & 2 & 13. Agriculturel Production Status & \\
\hline & \multirow{4}{*}{$\begin{array}{l}\text { 13.1 Density of } \\
\text { plant } \\
\text { production } \\
\text { planting area in } \\
\text { micro-basins }\end{array}$} & 4001> da cultivation area & 4 \\
\hline & & 2001-4000 da cultivation area & 3 \\
\hline & & 1001-2000 da cultivation area & 2 \\
\hline & & 100-1000 da cultivation area & 1 \\
\hline & & \multicolumn{2}{|l|}{ 14. Animal Production Status } \\
\hline & \multirow{4}{*}{$\begin{array}{l}\text { 14.1 The total } \\
\text { amount of } \\
\text { cattle and ovine } \\
\text { in micro-basins }\end{array}$} & $3501>$ animals & 4 \\
\hline & & 2001-3500 animals & 3 \\
\hline & & $1001-2000$ animals & 2 \\
\hline & & $300-1000$ animals & 1 \\
\hline \multirow{3}{*}{$\begin{array}{l}\text { 5- TOURISTIC } \\
\text { INFRASTRUCTURE } \\
\text { OPPORTUNITIES }\end{array}$} & & \multicolumn{2}{|c|}{$\begin{array}{l}\text { 15- Availability of Drinking Water, Electricity, } \\
\text { Communication Tools }\end{array}$} \\
\hline & 15.1 Presence & Availability of infastructure & 4 \\
\hline & $\begin{array}{l}\text { of drinking } \\
\text { water, }\end{array}$ & $\begin{array}{l}\text { Availability of other } \\
\text { infrastructure elements that the }\end{array}$ & 3 \\
\hline
\end{tabular}




\begin{tabular}{|c|c|c|c|}
\hline & \multirow{2}{*}{$\begin{array}{l}\text { electricity, } \\
\text { communication } \\
\text { tools in micro- } \\
\text { basins }\end{array}$} & $\begin{array}{l}\text { internet and mobile phone signal } \\
\text { cannot reach }\end{array}$ & \\
\hline & & Microbasin without infastructure & 1 \\
\hline \multirow{9}{*}{$\begin{array}{c}\text { 6- LEVEL OF } \\
\text { ORGANIZATION } \\
\text { AND SOCIAL } \\
\text { INTERACTION }\end{array}$} & \multicolumn{3}{|c|}{\begin{tabular}{l|l}
$16-$ Population Density \\
\end{tabular}} \\
\hline & \multirow{3}{*}{$\begin{array}{l}\text { 16.1 Population } \\
\text { density per sqm } \\
\text { in micro-basins }\end{array}$} & 1,24-1,84 popu./km2 & 4 \\
\hline & & $0,63-1,23$ popu. $/ \mathrm{km} 2$ & 3 \\
\hline & & $0,01-0,62$ popu. $/ \mathrm{km} 2$ & 2 \\
\hline & \multicolumn{3}{|c|}{\begin{tabular}{l|l}
17. Level of Organization \\
\end{tabular}} \\
\hline & \multirow[b]{2}{*}{$\begin{array}{l}17.1 . \\
\text { Union- } \\
\text { cooperative } \\
\text { containing } \\
\text { micro-basins }\end{array}$} & Union cooperative & 4 \\
\hline & & Non-union cooperative & 1 \\
\hline & & \multicolumn{2}{|l|}{ 18. Perception of Ecotourism } \\
\hline & $\begin{array}{l}\text { 18.1 Locals' } \\
\text { view of } \\
\text { ecotourism }\end{array}$ & Positive & 4 \\
\hline
\end{tabular}

*Scores are given in accordance with the literature review on the subject

With AHP Technique, weight scores of evaluation parameters regarding importance and priorities were determined. In the AHP process; firstly, a total of 12 people were selected from among the professions related to the subject of the study (Landscape architect, Urban Regional Planner, Forest Engineer, Geographer); after a hierarchical diagram was defined with criteria and sub-criteria, comparison matrices were prepared to indicate the relative importance or effect of a factor. After a hierarchical diagram was defined with criteria and sub-criteria, comparison matrices were prepared to indicate the relative importance or effect of a factor (Table 6).

Table 6. Analytical Hierarchy Process (AHP) Value Scale (Saaty, 2008)

\begin{tabular}{|c|l|}
\hline Value scale $(\mathrm{n})$ & \multicolumn{1}{c|}{ Definition } \\
\hline 1 & Both elements are equally important \\
\hline 3 & One element is slightly more important than the other \\
\hline 5 & One element is more important than the other \\
\hline 7 & One element is far more important than the othe \\
\hline 9 & One element is absolutely more important than the other \\
\hline $2,4,6,8$ & The mean between two adjacent value consideration \\
\hline
\end{tabular}

The forms regarding the evaluation criteria expected to be evaluated were sent to the experts via email. A total of 6 evaluations were made in which the experts evaluated the main topics and rated the factors under the main heading. The scores received were evaluated with the help of Expert Choice 
program. Samples with a coefficient of consistency less than 0.1 were evaluated, and a total of 8 evaluations were obtained. These 8 evaluations of 8 specialists were collected with arithmetic mean, and the factor coefficient was obtained (100 criteria of the factor coefficients were evaluated) and the factors were obtained by sharing them to the analyses to which they belong. The specified factor scores were entered into the database in ArcGis program (Table 7).

Table 7. Determination of the relative criterion weight

\begin{tabular}{|c|c|c|c|c|c|c|c|c|c|c|}
\hline & 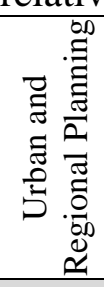 & 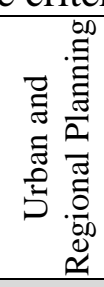 & 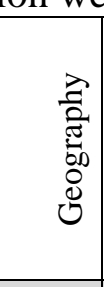 & 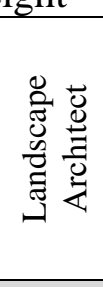 & 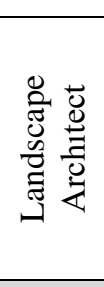 & 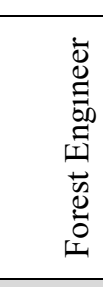 & 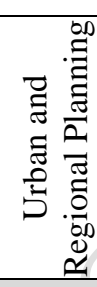 & 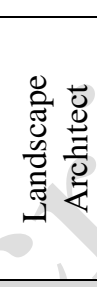 & 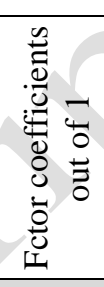 & 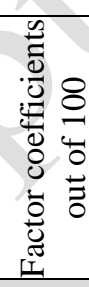 \\
\hline Accessibility to Roads & 0.154 & 0.031 & 0.059 & 0.043 & 0.004 & 0.015 & 0.019 & 0.006 & 0.041 & 4,1 \\
\hline Accessibility to Residential Areas & 0.154 & 0.031 & 0.044 & 0.043 & 0.021 & 0.015 & 0.022 & 0.014 & 0.043 & 4,3 \\
\hline Access to Ecotourism Resources & 0.154 & 0.031 & 0.197 & 0.043 & 0.021 & 0.015 & 0.004 & 0.034 & 0.062 & 6,2 \\
\hline Bioclimatic comfort & 0.01 & 0.076 & 0.062 & 0.025 & 0.083 & 0.011 & 0.062 & 0.083 & 0.052 & 5,2 \\
\hline Variety of Topographic Structures & 0.061 & 0.039 & 0.036 & 0.072 & 0.169 & 0.046 & 0.216 & 0.17 & 0.101 & 10,1 \\
\hline Aspect & 0.025 & 0.032 & 0.005 & 0.021 & 0.021 & 0.026 & 0.05 & 0.03 & 0.026 & 2,6 \\
\hline Forest Presence & 0.055 & 0.064 & 0.011 & 0.138 & 0.119 & 0.2 & 0.089 & 0.102 & 0.097 & 9,7 \\
\hline Water Presence & 0.055 & 0.064 & 0.016 & 0.138 & 0.066 & 0.2 & 0.078 & 0.102 & 0.090 & \\
\hline Soil Characteristics & 0.025 & 0.022 & 0.01 & 0.02 & 0.029 & 0.067 & 0.018 & 0.016 & 0.026 & 2,6 \\
\hline Folkloric Values & 0.02 & 0.076 & 0.062 & 0.113 & 0.042 & 0.071 & 0.011 & 0.05 & 0.056 & 5,6 \\
\hline $\begin{array}{l}\text { Historical- archeol } \\
\text { Structures }\end{array}$ & 0.061 & 0.076 & 0.055 & 0.113 & 0.163 & 0.071 & 0.11 & 0.109 & 0.095 & 9,5 \\
\hline Local Architecture & 0.061 & 0.076 & 0.032 & 0.113 & 0.047 & 0.071 & 0.051 & 0.028 & 0.060 & \\
\hline Traditional Life Culture & 0.02 & 0.076 & 0.059 & 0.038 & 0.047 & 0.071 & 0.023 & 0.036 & 0.046 & 4,6 \\
\hline Agricultural Production Status & 0.023 & 0.066 & 0.031 & 0.014 & 0.039 & 0.044 & 0.056 & 0.057 & 0.041 & 4,1 \\
\hline Animal Production Status & 0.005 & 0.066 & 0.062 & 0.014 & 0.039 & 0.044 & 0.056 & 0.057 & 0.043 & 4,3 \\
\hline $\begin{array}{l}\text { Availability of Drinking Water, } \\
\text { Electricity, Communication Tools }\end{array}$ & 0.061 & 0.057 & 0.075 & 0.025 & 0.042 & 0.018 & 0.067 & 0.016 & 0.045 & 4,5 \\
\hline Population Density & 0.008 & 0.013 & 0.03 & 0.003 & 0.004 & 0.013 & 0.007 & 0.042 & 0.015 & 1,5 \\
\hline Level of Organization & 0.023 & 0.048 & 0.055 & 0.013 & 0.014 & 0.005 & 0.043 & 0.029 & 0.029 & 2,9 \\
\hline Perception of Ecotourism & 0.023 & 0.057 & 0.1 & 0.013 & 0.028 & 0.001 & 0.017 & 0.02 & 0.032 & 3,2 \\
\hline
\end{tabular}

After all the values were processed and the relevant maps were created, the synthesis pad was created with Overlay Analysis (Mc Harg, 1969). In the synthesis map where all the studies are combined, priority areas for ecotourism are determined for Kofçaz.

Stage IV: At this stage, various strategies based on protection, planning, development and management have been produced for rural development-oriented ecotourism. In the development of 
relevant strategies, top scale plans and documents (Anonymous, 2007; 2013; 2014; 2018a; 2018b; 2019a; 2019b; 2020) and studies by Crisman et al. (2009), Kiper et al. (2015) and Kiper (2017) were effective. Strategy and objectives, taking into account the economic, ecological advantages of the workplace and the rapid rural evaluation method, which addresses the expectations of stakeholders, it was ensured that rural settlements were capable of maintaining and improving their original character.

\section{Results and Discussion}

Taking into account the factors, sub-units and evaluation criteria selected within the framework of ECOS method, analysis was made and thematic maps were created in the GIS environment using Arc GIS 10.2 software and accordingly 3D Analyst and Spatial Analyst modules. The evaluations of the analyses are presented below.

Accessibility refers to accessing the objectives and are the main indicators of location and distance. Within the scope of the study and the ECOS method, 18 criteria based on 3 basic, 5 sub-criteria and sub-criteria were evaluated and mapped in the GIS environment (Table 8, Figure 3, Figure 4, Figure 5, Figure 6, Figure 7).

Table 8. Accessibility criteria analysis results

\begin{tabular}{|l|l|l|c|c|}
\hline Criteria & Source & $\begin{array}{l}\text { Number of } \\
\text { Optimal } \\
\text { microcatchments }\end{array}$ & $\begin{array}{l}\text { Number of } \\
\text { available } \\
\text { microcatchments }\end{array}$ & $\begin{array}{l}\text { Figure } \\
\text { number }\end{array}$ \\
\hline 1.1 Access to roads & 65 & & Figure 3 \\
\hline 1.2 Access density to roads & $\begin{array}{l}\text { Jones et al., 1997, } \\
\text { Uzun et al., 2015 }\end{array}$ & 4 & 15 & Figure 4 \\
\hline $\begin{array}{l}2.1 \text { Access from micro-basin to } \\
\text { settlements }\end{array}$ & $\begin{array}{l}\text { Figure 5 } \\
\text { 3.1 Access from micro-basin to } \\
\text { recreational activity (m) }\end{array}$ & 80 & 49 & Figure 16 \\
\hline $\begin{array}{l}3.2 \text { Access from micro-basin to } \\
\text { water resources (m) }\end{array}$ & Kiper et al., 2015 & 125 & 20 & Figure 17 \\
\hline
\end{tabular}




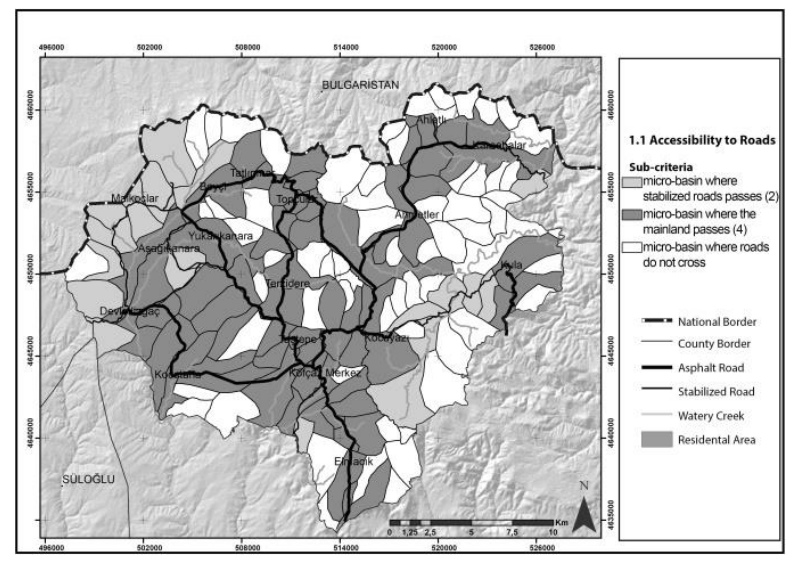

Figure 3. Access to roads

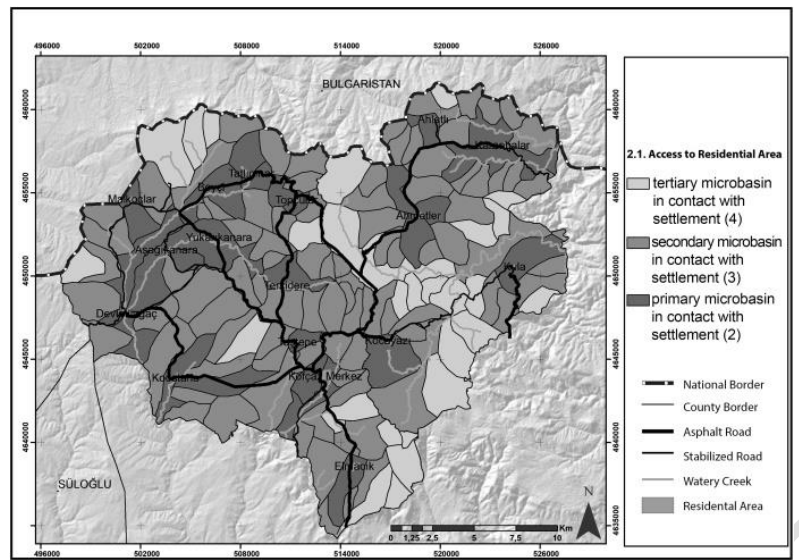

Figure 5. Access from micro-basin to settlements

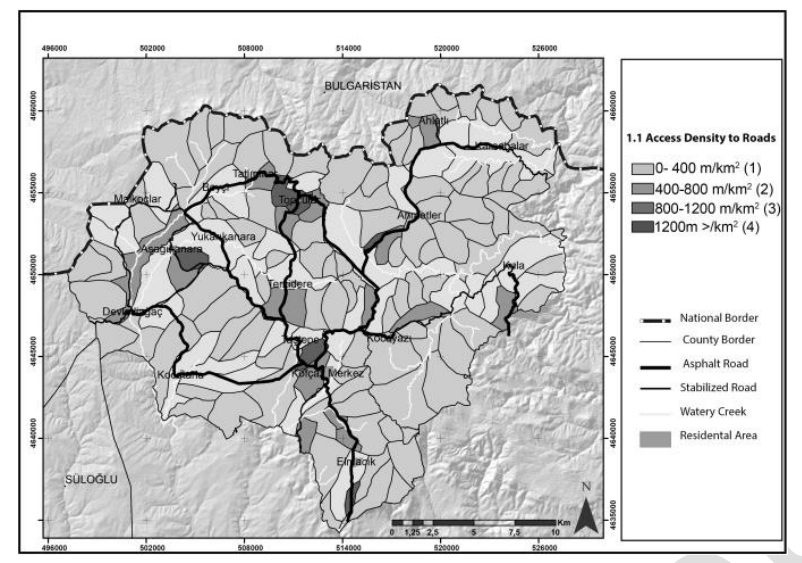

Figure 4. Access density to roads

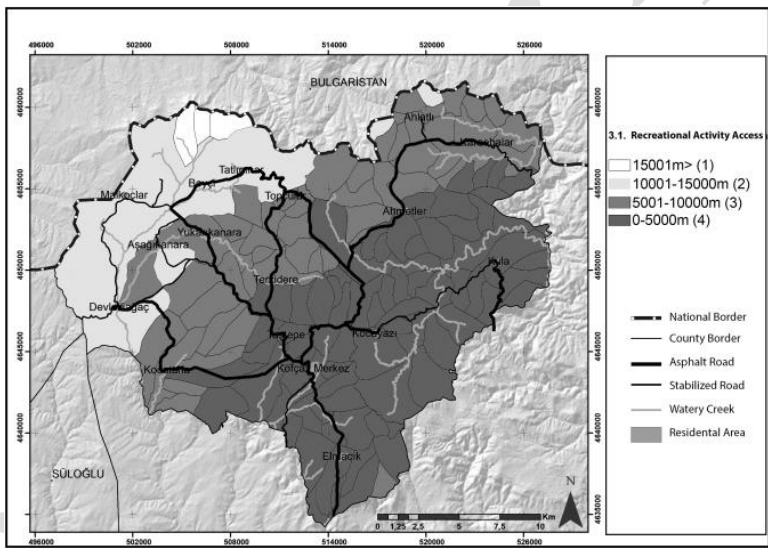

Figure 6. Access from micro-basin to recreational activity $(\mathrm{m})$

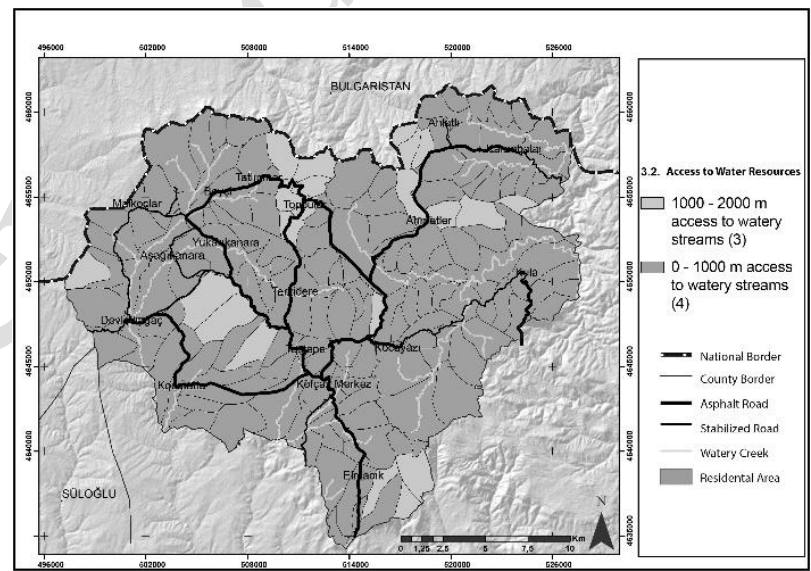

Figure 7. Access from micro-basin to water resources (m)

Natural Landscape Charms of Ecotourism Source: Ecological resource is the main source of tourism for ecotourism and is an important value that increases the attractiveness of tourism (Mai and Smith, 2015; Choi et al., 2021). Within the scope of the study and ECOS method, 5 basic and 29 sub-criteria were evaluated under this heading and mapped in the GIS environment (Table 9). 
Bioclimatic comfort has an effective role in the development of ecotourism opportunities. A method of biocyclical comfort analysis was followed in the evaluation of climate parameters. Using the RayMan model for bioclimatic comfort analysis, PET calculation was performed and in Arc GIS 10.2 software was interpolated with Inverse Distance Weighted (IDW) method. In RayMan, the average monthly temperature, relative humidity and wind measurement values for the 17 climate stations in the Kirklareli province borders were used as obtained from the General Directorate of Meteorology of the Ministry of Agriculture and Forestry for many years between 1980 and 2018. The resulting maps were classified according to the comfort zones that determine bioclimatic comfort, and bioclimatic comfort map was created (Figure 8).

Topographic structure diversity; three main criteria and 11 sub-criteria were covered as groups of height, slope, and exposure. This creates interesting possibilities for ecotourism (Figure 9).

Table 9. Natural criteria analysis results

\begin{tabular}{|l|l|l|l|c|}
\hline Criteria & Source & $\begin{array}{l}\text { Number of } \\
\text { Optimal } \\
\text { microcatchments }\end{array}$ & $\begin{array}{l}\text { Number of } \\
\text { available } \\
\text { microcatchments }\end{array}$ & $\begin{array}{l}\text { Figure } \\
\text { number }\end{array}$ \\
\hline 4.1 Bioclimatic comfort & $\begin{array}{l}\text { Javan and Malazadeh, } \\
2013 ; \text { Ramazani } \\
\text { Gourbi, 2010; Gourab1 } \\
\text { and Palic, 2012 }\end{array}$ & 90 & Figure 8 \\
\hline 5.1 Height group variety & & 37 & & Figure 9 \\
\hline 5.2 Average slope (\%) & Topay, 2003 & 145 & Figure 10 \\
\hline 5.3 Aspect density & & 58 & & Figure 11 \\
\hline 6.1 Forest stand canopy cover & & 85 & Figure 12 \\
\hline $\begin{array}{l}\text { 7.1 Stream density in forest areas in } \\
\text { microbasins }\end{array}$ & $\begin{array}{l}\text { Jones et al., 1997; Uzun } \\
\text { et al., 2015 }\end{array}$ & 27 & Figure 13 \\
\hline 8.1 Erosion in micro-basin & Uzun et al., 2015 & 45 & & Figure 14 \\
\hline $\begin{array}{l}\text { 8.2 Landuse capability classes in } \\
\text { micro-basins }\end{array}$ & Anonymous, 2017 & 15 & 108 & Figure 15 \\
\hline
\end{tabular}

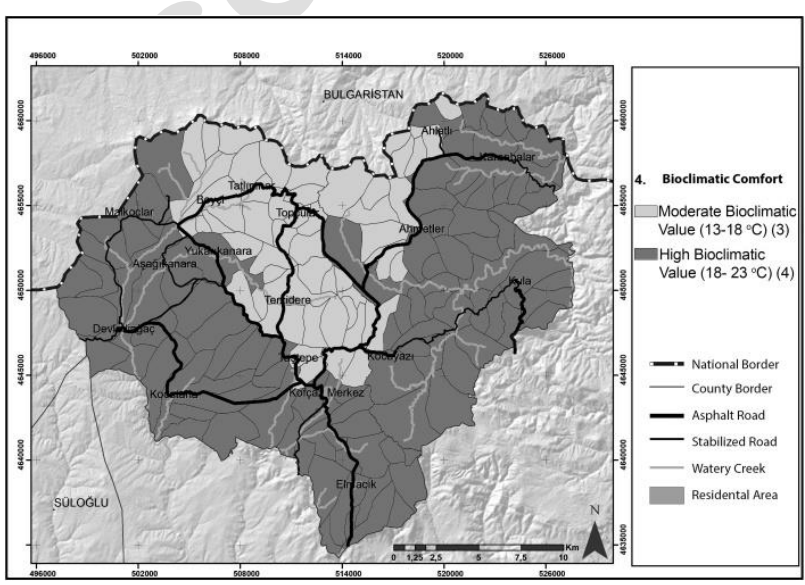

Figure 8. Bioclimatic comfort

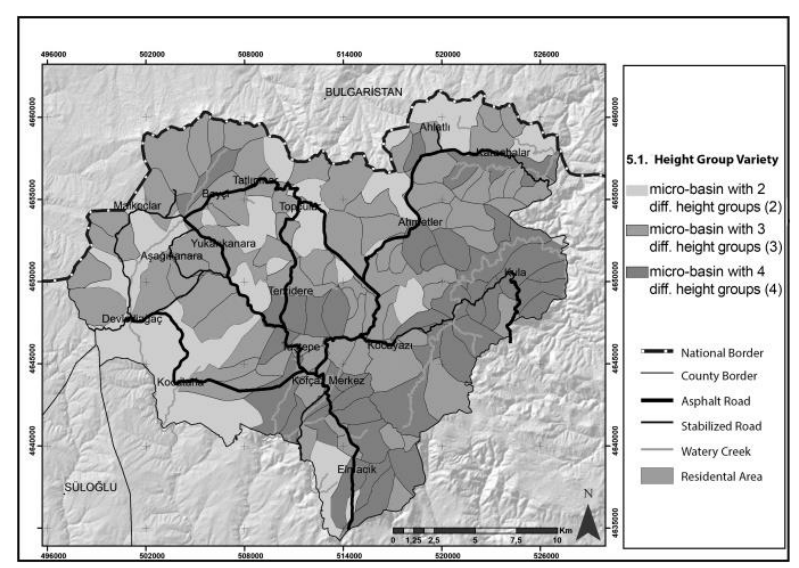

Figure 9. Height group variety 


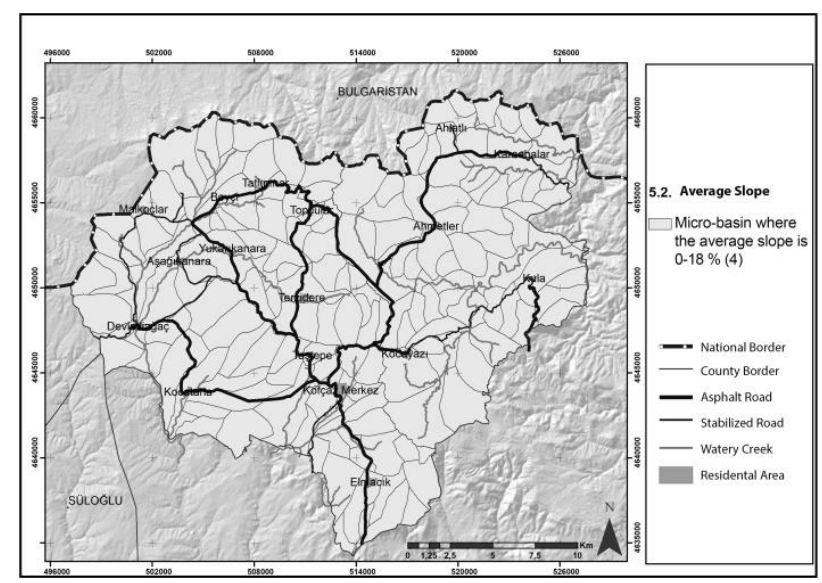

Figure 10. Average slope

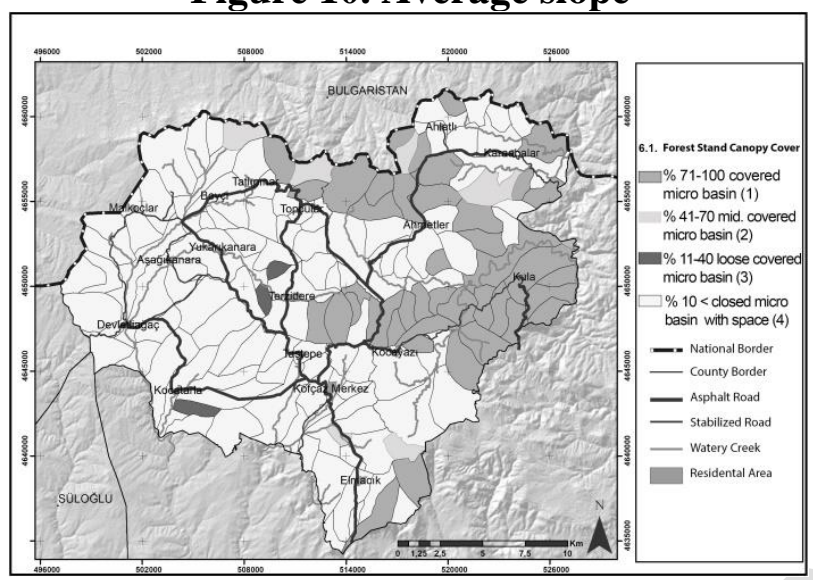

Figure 12. Forest stand canopy cover

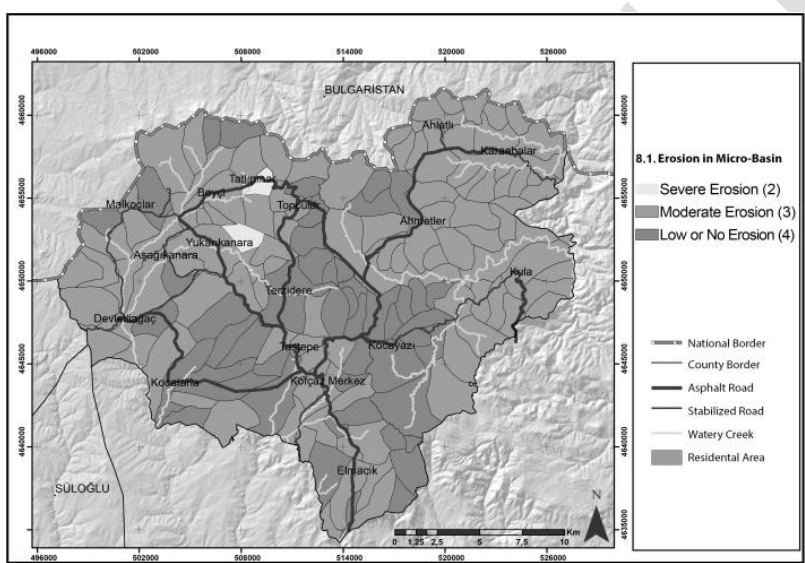

Figure 14. Erosion in microbasin

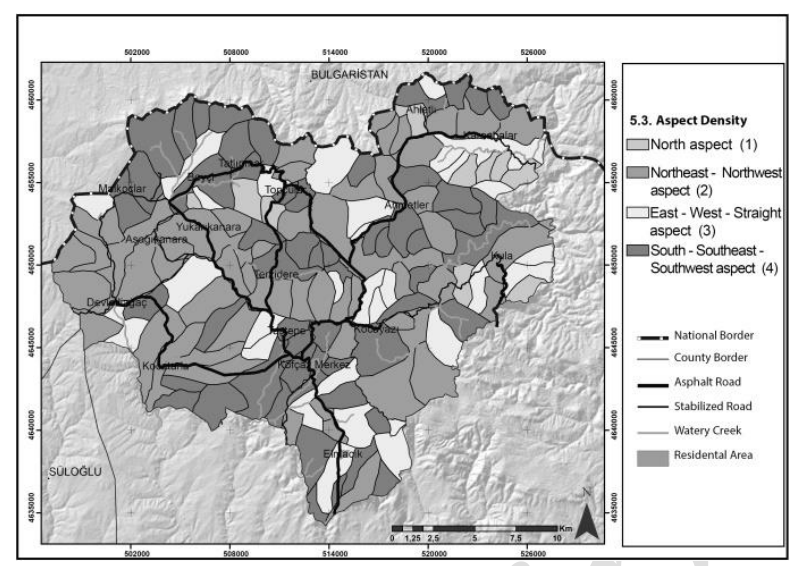

Figure 11. Aspect density

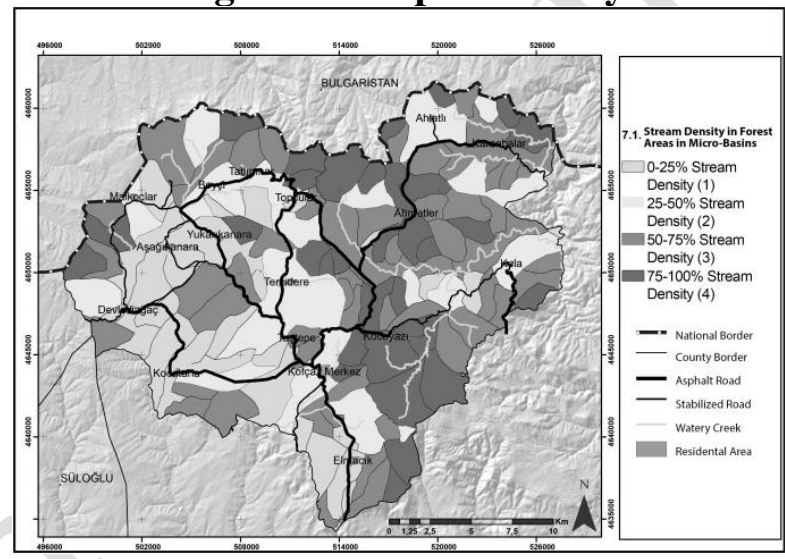

Figure 13. Stream density in forest areas

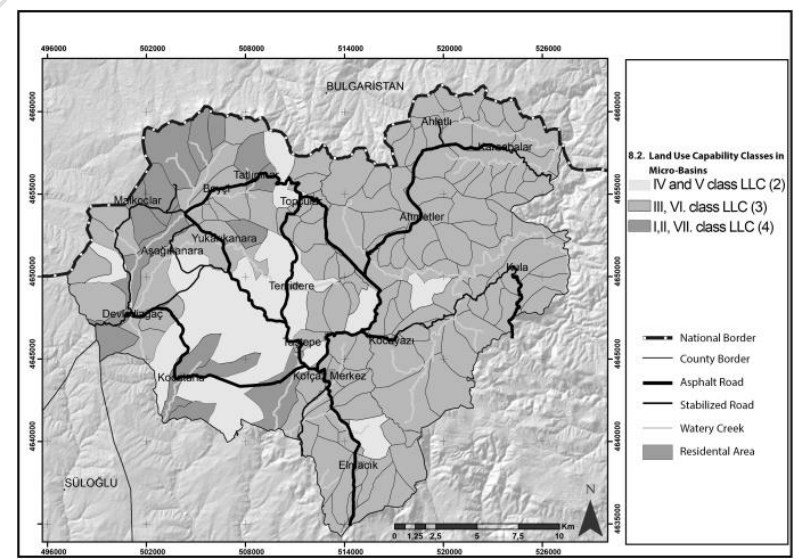

Figure 15. Landuse capability classes

Cultural Landscape Attractiveness Values of Ecotourism Source: Cultural landscape attractiveness is effective in settlement formation, development of economic structure and shaping of landscape identity (Bahçe, 2009; Erdem, 2012). Within the scope of the study; 4 basic and 7 sub-criteria were evaluated under this title, and mapped in a GIS environment (Table 10; Figure 16, Figure 17, Figure 18, Figure 19). 
Table 10. Culturel landscape attractiveness criteria analysis results

\begin{tabular}{|l|l|l|}
\hline Criterion & Resource & Evaluation and Figure no. \\
\hline $\begin{array}{l}\text { 9.1 Availability of folkloric } \\
\text { values in micro-basins }\end{array}$ & Gültekin, 2014 & $\begin{array}{l}\text { While the micro-basin in which Kocayazi is located } \\
\text { was determined as the most suitable, Kofçaz center, } \\
\text { Kula, Ahmetler, Elmacik, Aşağlkanara and micro- } \\
\text { basins were determined as suitable micro-basins } \\
\text { (Figure 16) }\end{array}$ \\
\hline $\begin{array}{l}\text { 10.1 Accessibility to historical } \\
\text { archaeological values in micro- } \\
\text { basins }\end{array}$ & $\begin{array}{l}\text { The micro-basins where Devletliağaç, Taştepe, } \\
\text { Kocayazl, Ahmetler, Ahlatl, Karaabalar, Malkoçlar, } \\
\text { Aşağlkanara, Yukarikanara, Taştepe, Tatlipınar } \\
\text { Kofçaz centers are located were determined as the } \\
\text { most suitable and appropriate ones (Figure 17). }\end{array}$ \\
\hline $\begin{array}{l}\text { 11.1 Micro-basins with local } \\
\text { architectural structures }\end{array}$ & $\begin{array}{l}\text { Micro-basins where Kocayazl, Kula, Ahmetler, } \\
\text { Karaabalar, Ahlatl, Topçular, Beyci, Malkoçlar and } \\
\text { Elmacik settlements are located received the most } \\
\text { suitable value in terms of ecotourism (Figure 18). }\end{array}$ \\
\hline $\begin{array}{l}\text { 12.1 Diversity of traditional life } \\
\text { culture in micro-basins }\end{array}$ & $\begin{array}{l}\text { Micro-basins containing 16 rural settlements where } \\
\text { Amuca, Gacal, Pomak and Bosniak culture are located } \\
\text { were determined as most suitable for ecotourism } \\
\text { activities (Figure 19) }\end{array}$ \\
\hline
\end{tabular}

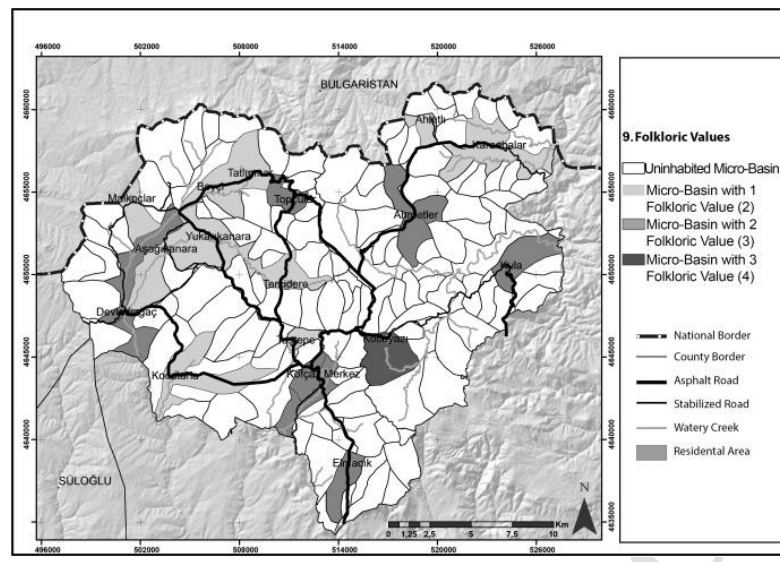

Figure 16. Availability of folkloric values in micro-basins

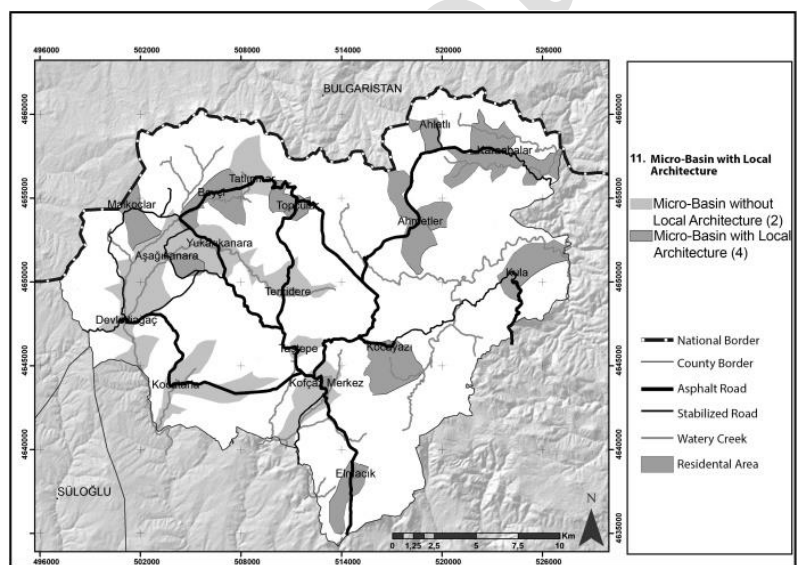

Figure 18. Micro-basins with local architectural structures

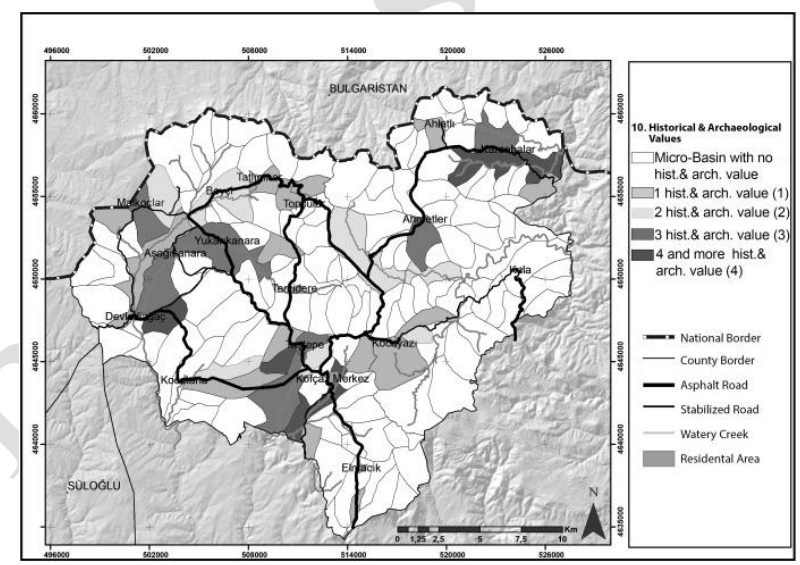

Figure 17. Accessibility to historical archaeological values in micro-basins

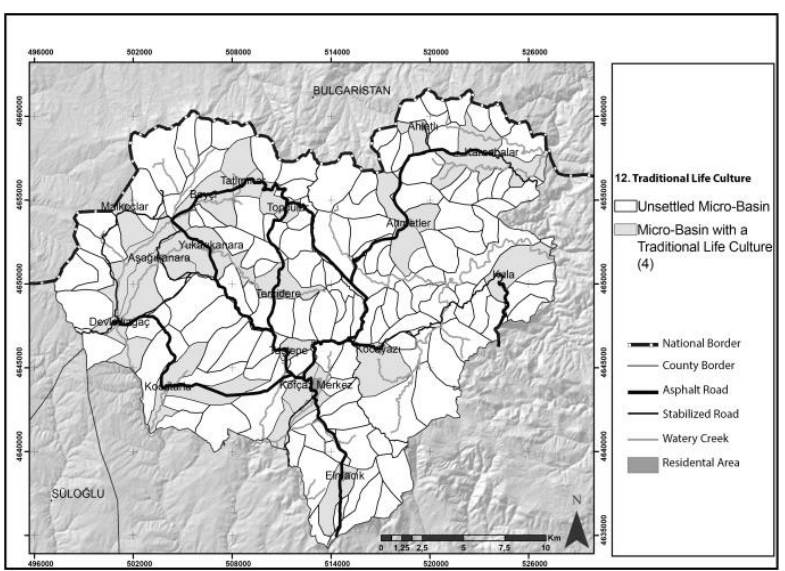

Figure 19. Diversity of traditional life culture in micro-basins 
Economic structure diversity: Economic structure forms are the local characteristics of the relevant region and are an important resource value for ecotourism activities. In the study; 2 sub-criteria and 8 parameters related to the diversity of economic structure were discussed (Table 11).

Status of tourist infrastructure facilities: While infrastructure opportunities in an area where ecotourism development is envisaged are affecting the demand for the region, they are important for increasing accessibility to ecotourism opportunities and continuity of ecotourism (Goeldner and Ritchie, 2002) (Table 11).

Level of organization and social interaction of ecotourism source: In the study; 3 sub-criteria and 6 parameters based on sub-criteria were discussed under this heading (Table 11). All three criteria were values that influence and influence ecotourism.

Table 11. Cultural landscape attractiveness values, economic structure, touristic infrastructure opportunities, level of organization and social interaction criteria of ecotourism source

\begin{tabular}{|c|c|c|}
\hline Criterion & Resource & Evaluation and Figure no. \\
\hline $\begin{array}{l}\text { 13.1 Density of plant production } \\
\text { planting area in micro-basins }\end{array}$ & $\begin{array}{l}\text { Çevik and Tekinel, 1998; } \\
\text { Sanır, 2000; Zaman, 2010; } \\
\text { Arıbaş, 2010; Tekeli, 2016 }\end{array}$ & $\begin{array}{l}\text { While the settlements of Kofçaz-Center, } \\
\text { Elmacık, Ahmetler, Aşağıkanara, } \\
\text { Yukarıkanara, Malkoçlar, Terzidere and } \\
\text { Karaabalar received } 4 \text { points, the micro- } \\
\text { basins of Ahlatlı, Tatlıpinar and Taştepe } \\
\text { settlements received } 3 \text { points (Figure 20). }\end{array}$ \\
\hline $\begin{array}{l}\text { 14.1 The total amount of cattle } \\
\text { and ovine in micro-basins }\end{array}$ & $\begin{array}{l}\text { Çevik and Tekinel, 1998; } \\
\text { Sanır, 2000; Zaman, 2010; } \\
\text { Arıbaş, } 2010\end{array}$ & $\begin{array}{l}\text { The settlements of Aşağıkanara, } \\
\text { Yukarıkanara, Devletliağaç, Terzidere and } \\
\text { Kofçaz-centeri received } 4 \text { points, while the } \\
\text { micro-basins of Ahmetler, Kocayazi, } \\
\text { Beyci, Elmacik and Taştepe received } 3 \\
\text { points. (Figure 21). }\end{array}$ \\
\hline $\begin{array}{l}\text { 15.1 Presence of drinking water, } \\
\text { electricity, communication tools } \\
\text { in micro-basins }\end{array}$ & $\begin{array}{l}\text { Neth, 2008; Türker, 2013; } \\
\text { Gültekin, 2014; Kiper et al., } \\
\text { 2015; Salıc1, 2018; Rudianto } \\
\text { et al., 2019; Alam, 2019, } \\
\text { Kabataş, 2020; Yiğit, 2020 }\end{array}$ & $\begin{array}{l}\text { All micro-basins with rural settlements } \\
\text { received a score of } 4 \text { points (Figure } 22 \text { ). }\end{array}$ \\
\hline $\begin{array}{l}\text { 16.1 Population density per sqm } \\
\text { in micro-basins }\end{array}$ & $\begin{array}{l}\text { Şahin, 2009; Tümertekin } \\
\text { and Özgüç, 2015). }\end{array}$ & $\begin{array}{l}\text { Micro-basins in Kofçaz central settlement } \\
\text { received a value of } 4 \text { points (Figure 23). }\end{array}$ \\
\hline $\begin{array}{l}17.1 \text { Union-cooperative } \\
\text { containing } \\
\text { micro-basins }\end{array}$ & Anonymous, 2018a & $\begin{array}{l}\text { The micro-basins of Kofçaz center, Ahlatl, } \\
\text { Karaabalar, Ahmetler, Kocayazi and Kula } \\
\text { received a score of } 4 \text { points (Figure 24). }\end{array}$ \\
\hline 18.1 Locals' view of ecotourism & & $\begin{array}{l}\text { All micro-basins with rural settlements in } \\
\text { Kofçaz received a score of } 4 \text { points (Figure } \\
25 \text { ). }\end{array}$ \\
\hline
\end{tabular}




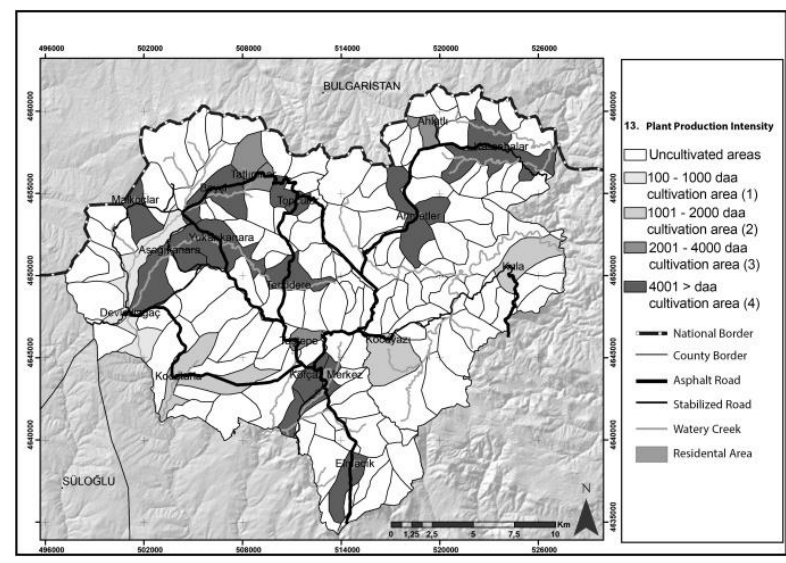

Figure 20. Density of plant production planting area in micro-basins

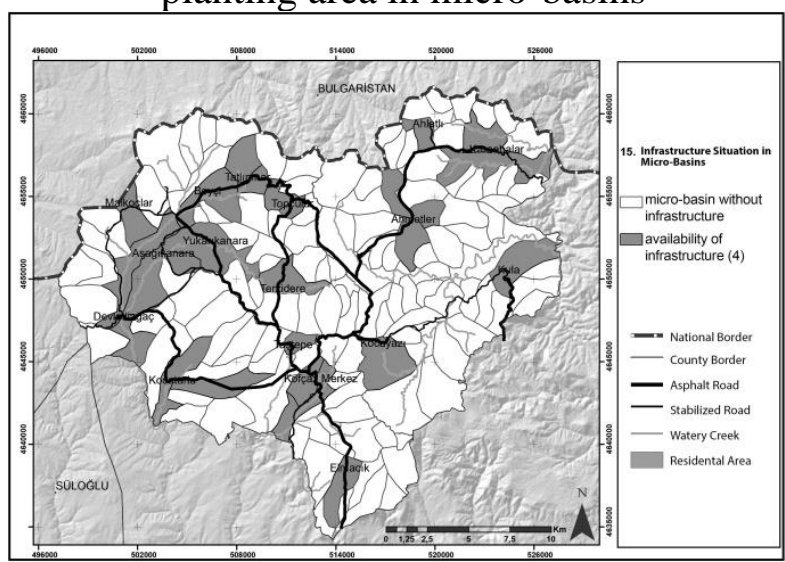

Figure 22. Presence of drinking water, electricity, communication tools in microbasins

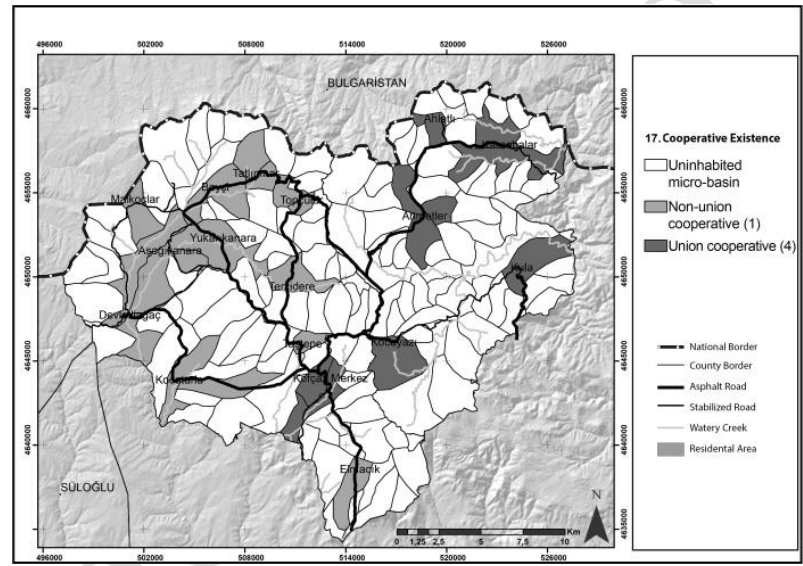

Figure 24. Union-cooperative containing micro-basins

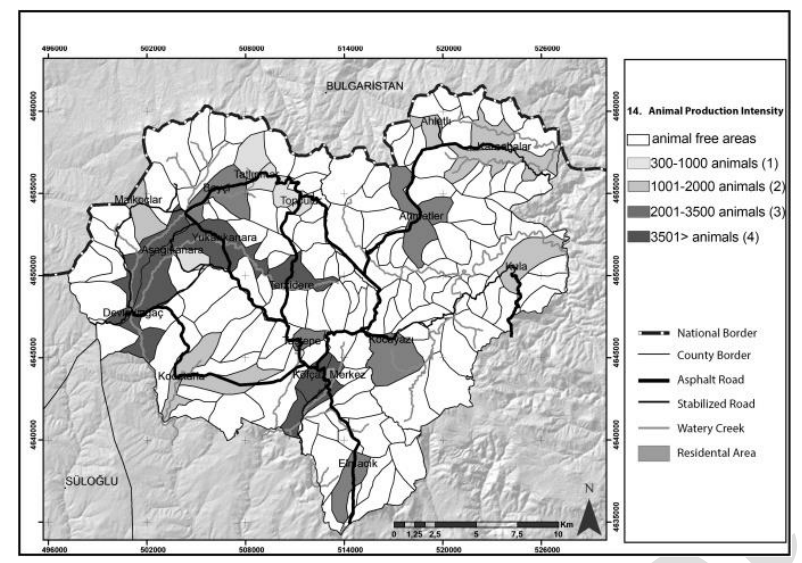

Figure 21. The total amount of cattle and ovine in micro-basins

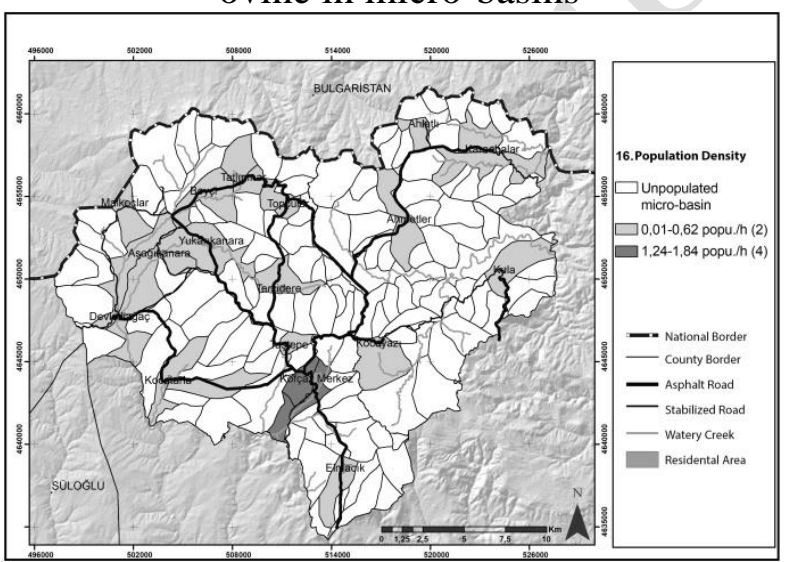

Figure 23. Population density per sqm in micro-basins

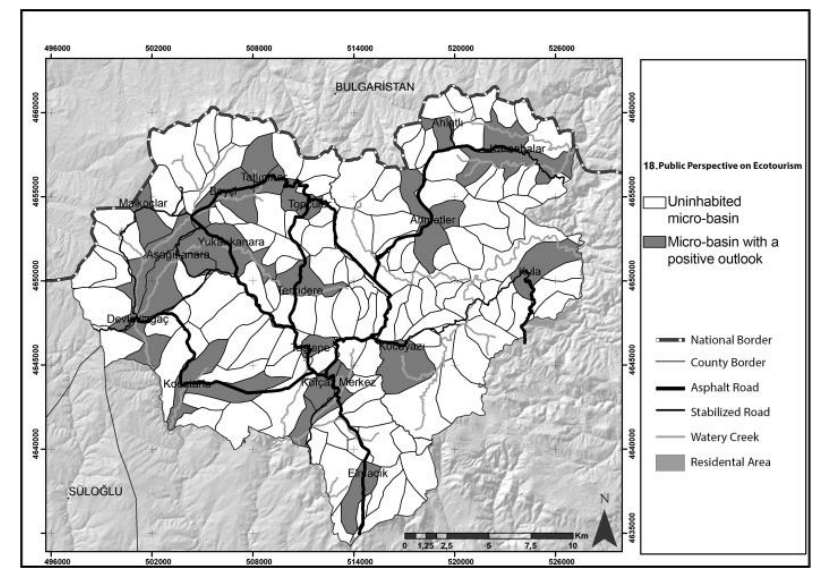

Figure 25. Locals' view of ecotourism

\section{Conclusions}

Within the framework of ECOS method developed in the study, the criteria determined within the framework of the 145 micro-basins were applied to the conformity maps created by overlapping the potential field usage map for ecotourism (Figure 26). In the preparation of ecotourism conformity 
maps of data layers, the conformity values of the criteria and the weight coefficients determined by AHP technique were based. Afterwards, the total scores for ecotourism compliance of each microbasin in terms of 23 criteria were calculated with the collection of points for each micro-basin. The sub-criterion scores obtained in the chart of the method were multiplied by the following weight coefficients obtained as a result of the AHP process, resulting in scores between 184 and 452 for 145 micro-basins. The difference in points is divided into 4 equal parts, sorted according to the value ranges and the number of micro-basins were determined (Table 11).

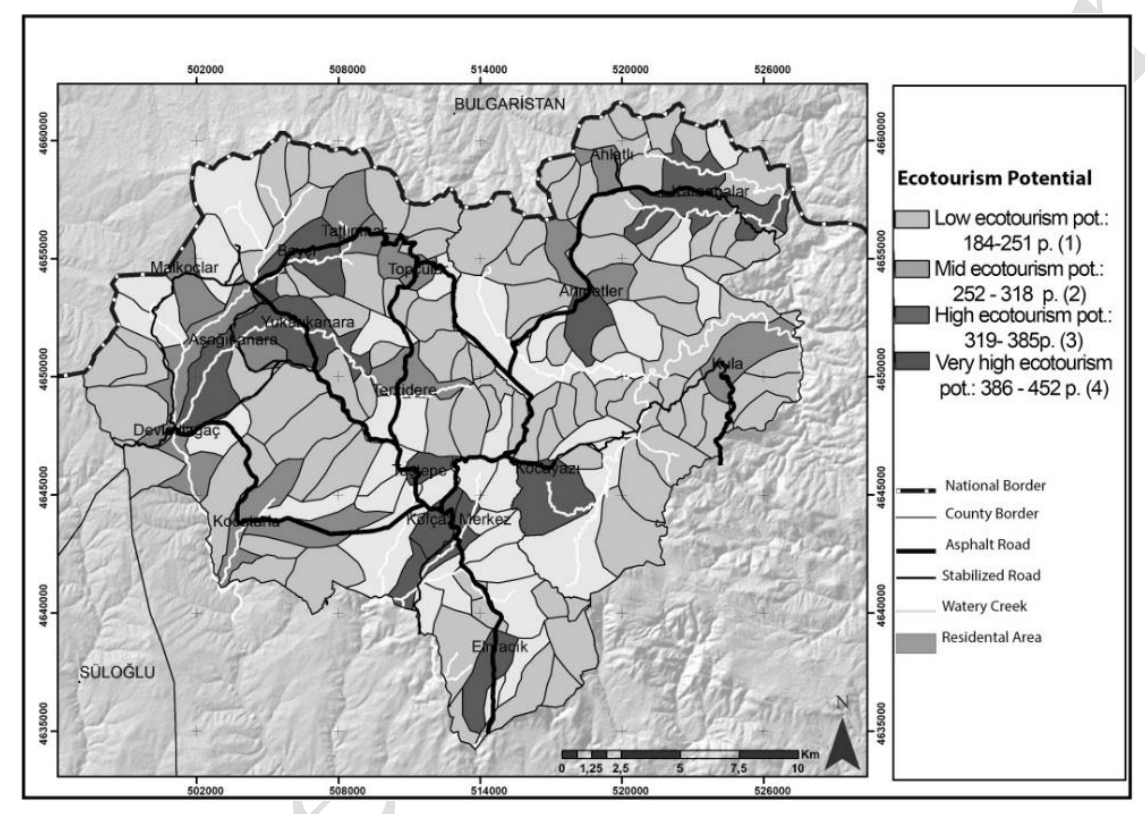

Figure 26. Ecotourism suitability of micro-basin

Table 11. Value range of ecotourism opportunity

\begin{tabular}{|l|l|c|}
\hline \multicolumn{1}{|c|}{ Value range of ecotourism opportunity } & Total \\
\hline between 184-151 & Low (Microcatchments less suitable for ecotourism) & 89 \\
\hline between aras1 & Mid (Microcatchments mid suitable for ecotourism) & 31 \\
\hline between 319-385 & High (Microcatchments suitable for ecotourism) & 15 \\
\hline between 386-452 & $\begin{array}{l}\text { Very High (Microcatchments very suitable for } \\
\text { ecotourism) }\end{array}$ & 10 \\
\hline
\end{tabular}

In addition, various strategies have been developed for the development of areas with less appropriate ecotourism opportunities in the field of study. Strategies were discussed under 6 basic headings aimed at improving ecotourism within the framework of "spatial", "participatory" and "sustainability" approaches. Related strategies; planning, promotion, entrepreneurship, education and cooperationorganization were shaped in line with the main themes (Table 12). 
Table 12. Ecotourism development strategies

\begin{tabular}{|l|l|l|}
\hline Main theme & Strategy & Approach \\
\hline Planning & $\begin{array}{l}\text { Establishment of thematic development corridors for } \\
\text { ecotourism }\end{array}$ & $\begin{array}{l}\text { Spatial } \\
\text { Environmental }\end{array}$ \\
\cline { 2 - 3 } & Establishment of thematic development zones for ecotourism & \\
\hline Promotion & Creating an ecotourism image & Social, \\
Promotion, & $\begin{array}{l}\text { Diversification of economic activities specific to rural } \\
\text { Entrepreneurship }\end{array}$ & $\begin{array}{l}\text { Economic } \\
\text { Participatory }\end{array}$ \\
\hline Cooperation-Organization & $\begin{array}{l}\text { Ensuring coordination and cooperation among stakeholders for } \\
\text { ecotourism and improving organization opportunities }\end{array}$ & $\begin{array}{l}\text { Social, } \\
\text { Economic } \\
\text { Participatory }\end{array}$ \\
\hline $\begin{array}{l}\text { Education } \\
\text { Entrepreneurship }\end{array}$ & $\begin{array}{l}\text { Leadership of local people in ecotourism and raising awareness } \\
\text { for tourism }\end{array}$ & $\begin{array}{l}\text { Social, } \\
\text { Participatory }\end{array}$ \\
\hline
\end{tabular}

ECOS method was used to determine ecotourism development areas within the scope of the study.

The related method was developed by differentiating the content, criteria and scope from the ECOS method used in other similar studies. Likewise, in similar studies using ECOS method, evaluations were made based on surveys and expert opinions. Five innovative aspects of this study can be highlighted.

- In different studies, the potential of ecotourism determined at the level of settlements with ECOS method was realized at the level of micro-basins. The micro-basin-level GIS-based data set also contributes significantly to decision makers in managing the potential for that micro-basin and solving the problem of the micro-basin.

- In the study, the use of too many variables within the framework of 23 main and 73 sub-parameters, developed based on natural and cultural landscape attractiveness, accessibility, economic building attractiveness, level of organization with infrastructure possibilities and social interaction, has increased the sensitivity of the study. At the same time, it has ensured that ecological, economic, cultural and social criteria are evaluated together.

- In this study, the integration of bioclimatic comfort with ECOS method is also an innovative approach for recent studies.

- At the micro-basin level, based on space-based data, rapid rural evaluation techniques and participatory understanding of ecotourism strategies are constructed through spatialized data sets 
at the micro-basins level, making the relationship between space and decision processes concrete and applicable.

- Expert participation in determining the potential of ecotourism (AHP), local government and public opinions were reflected in the decisions in determining the strategy, and participation on different scales was reflected in the process.

As a result, spatialization of ECOS method at the micro-basin level in rural development-oriented ecotourism planning and development of ecotourism management strategies with a participatory process can be effective when done with an interdisciplinary stakeholder group of different scales. The fact that the method approach put forward can be used especially in ecotourism planning on the scale of basins and sub-basins or in studies to be carried out at the provincial level, and that the method is constructed in accordance with universal data sets in different countries, suggests that the method approach put forward by the study can be easily used by experts and decision makers working on the subject at national and international level.

\section{Acknowledgements}

This study was supported by the The Scientific and Technological Research Council of Turkey Rapid Support Project No. 1200155

\section{References}

Açiksöz, S., Görmüş, S., Karadeniz, N. (2010). Determination of ecotourism potential in national parks: Kure Mountains National Park, Kastamonu-Bartin, Turkey. Afr. J. Agri. Res., 5, 8, 589599. https://doi.org/10.5897/AJAR09.735

Ajlaoni, N. (2011). Ecotourism and cultural landscape conservation. Faculty of Engineering at Birzeit University, Masters Thesis, Palestina. http://hdl.handle.net/20.500.11889/1675

Alam, M. F. (2019). Strategi pengembangan wisata mangrove dengan pendekatan ecotourism opportunity spectrum di kawasan wisata mangrove exotic mengare kabupaten gresik. Sarjana thesis, Universitas Brawijaya. http://repository.ub.ac.id/id/eprint/170887. 
Alexander, S.E., Whitehouse, J.L. (2004). Challenges for balancing conservation and development through ecotourism: 1nsights and implications from two Belizean Case Studies.In F.D. Pineda, C.A Brebbia ve M.Mugica, Sustainable tourism. Southampton: WIT press.

Anonymous, (2007). Turkey's Tourism Strategy 2023 Action Plan. 2007. The Ministry of Culture and Tourism, Ankara. p. 60. ISBN 978-975-17-3281-1

Anonymous, (2009). 1/100.000 scaled Thrace Subregion Ergene Basin Revision Environment Planning, Ministery of Environmental and Forest, p. 490.

Anonymous, (2013). Tenth Development Plan (2014-2018). Ministry of Development, Ankara.

Anonymous, (2014). Thrace Regional Plan (2014-2023). Trakya Development Agency, https://www.trakyaka.org.tr/upload/Domain/trakyaka/trakya_ka_rev.pdf.

Anonymous, (2017). Regulation on the Protection and Use of Agricultural Lands. http://gis.nacse.org/rewab/docs/Regulation_on_Utilization_Protection_of_Agricultural_Lands_ 2003_Tu.pdf.

Anonymous, (2018a). Eleventh Development Plan (2019-2023) Rural Development Special Expertise Commission Report. Ministry of Development, p. 140, Ankara.

Anonymous, (2018b). Eleventh Development Plan (2019-2023) Tourism Special Expertise Commission Report. Ministry of Development, p. 140, Ankara.

Anonymous, (2019a). Eleventh Development Plan (2019-2023). Presidency of the Republic of Turkey presidency Strategy and Budget. p. 212, Ankara.

Anonymous, (2019b). National Smart City Strategy and Action Plan (2020-2023). Ministry of Environment and Urbanisation, p. 658, Ankara.

Anonymous, (2020). Turkey Spatial Strategy Plan Scoping Report. Ministry of Environment and Urbanisation, İstanbul Technical University, p.101, Ankara. 
Arıbaş, K. (2010). Settlement in Turkey. (Edit: H.Yazıcı, M.K. Koca), Turkey Geography and Geopolitics, Ankara: Pegem Publishing. ISSN:1304-0278

Ashok, S., Tewari, H.R., Behera, M.D., Majumdar, A. (2017). Development of ecotourism sustainability assessment framework employing Delphi, C\&I and participatory methods: a case study of KBR, West Sikkim, India. Tour Manag Perspect, 21:24-41. https://doi.org/10.1016/j.tmp.2016.10.005

Bahçe, A. S. (2009). Cultural (Heritage) Tourism Model In Rural Development. Dumlupınar University Journal of Social Sciences, 25, 1-12.

Bi, Y.L. (2005). Operationalising the Ecotourism Opportunity Spectrum (ECOS): Case examples from China. University of Otago Department Tourism, Masters Thesis. http://hdl.handle.net/10523/1298

Boyd, S. W. and Butler, R. W. (1996). Managing ecotourism: an opportunity spectrum approach. Tour. Manage., 17: 556-566. https://doi.org/10.1016/S0261-5177\%2896\%2900076-3

Crisman T.L., Takavakoglou V., Alexandridis T., Antonopoulos V. and Zalidis G. (2009). Rehabilitation of abandoned saltworks to maximize conservation, ecotourism and water treatment potential. Global NEST Journal, 11:1, 24-31.

Cengiz, A.E., Ayhan, C.K., Timur U. P. (2016). Method for determination of the potential for ecotourısm in the Canakkale Wars Gallıpolı Historıcal Area Canakkale, Turkey. Oxıdatıon Communications, 39,1, 641-660.

Cheia, G. (2013). Ecotourism: definition and concepts. Journal of Tourism, 15, 56-60. https://econpapers.repec.org/RePEc:scm:rdtusv:v:15:y:2013:i:15:p:56-60

Choi Y.E., Oh, C., Chon, J. (2021). Applying the resilience principles for sustainable ecotourism development: A case study of the Nakdong Estuary, South Korea.Tourism Management, 83, 111. https://doi.org/10.1016/j.tourman.2020.104237 
Collins, M.G., Steiner, F.R., Rushman, M.J. (2001). Landuse suitability analysis in the United States: historical development and promising technological achievements. Environmental Management, 28, 5, 611-621. https://doi.org/10.1007/s002670010247.

Çekül, (2012). Thrace uniting power: nature, water, culture strategy plan culture priority regional roadmaps. https://www.cekulvakfi.org.tr/proje/2012-yol-haritalari.

Çelik, Z. (2006). Developıng rural plannıng polıcıes in Turkey. Dokuz Eylul University Institute of Science and Technology, Urban and Regional Planning, Department of City Planning. PhD Thesis, İzmir.

Çevik, B. and Tekinel, O. (1998). Rural settlement technique. Adana: Çukurova University Publications.

Erdem, M. (2012). Evaluation matrix proposal for identification, preservation and development of landscape characteristics of rural settlements. İstanbul Technical University Graduate School of Natural and Applied Sciences Department of Landscape Architecture Doctoral Thesis, İstanbul.

Fagence, M. (2001). Strategies for developing ecotourism in Pacific Island Countries: Presentation to UNESCAP/SPTO seminar on sustainable development of ecotourism in Pacific Island Countries. School of Geography, University of Queensland Suva, Fiji.

Fattah, M., Candra, A.I., Tiwi N., U., (2021). Analyzing the position value of Mangrove Bee Jay Bakau Resort, Indonesia. Eco. Env. \& Cons. , 27, 367-371.

Forman, R.T.T. (1995). Land mosaics. The Ecology of Landscape and Region. Cambridge, UK: Cambridge University Press.

Gigović, L., Pamučar, D., Lukić, D., Marković, S. (2016). GIS-Fuzzy DEMATEL MCDA model for the evaluation of the sites for ecotourism development: A case study of "Dunavski ključ" region, Serbia. Land Use Policy, 58(1), 348-365. http://dx.doi.org/10.1016/j.landusepol.2616.07.030. 
Goeldner, R.C. and Ritchie, J.R.B. (2002). Tourism: principles, practices, philosophie. Canada: John Wiley\&Sons Inc., 9th edition, p.250.

Gourab1, B.R. and Palic, M. (2012). Recognition of monthly human bioclimatic comfort with tourism climate index In Ramsar, South-West Of Caspian Sea, Iran. AGD Landscape \& Environment. 6 ,1, 1-14. https://ojs.lib.unideb.hu/landsenv/article/view/2288

Görmüş, S. (2017). Determining ecotourism circulation corridors using GIS in. Küre Mountains National Park, Turkey. Current Trends in Science and Landscape Management, 121-138.

Gülçubuk B.,Yıldırak, N., Kızılaslan, N., Özer, D., Kan, M., Kepoğlu, A. (2016). Rural Development Approaches and Changing Policy. http://www.zmo.org.tr/resimler/ ekler /e443d6819ae22b2 _ek . pdf.

Gültekin, P. (2014). Landscape planning and rural development focused with ecotourism in Ugursuyu and Aksu watersheds. Düzce University Graduate School of Natural and Applied Sciences Department of Landscape Architecture Doctoral Thesis, Düzce.

Hardy, A., Pearson, L., Davidson, P., Kriwoken, L. (2014). Social analysis of sustainable tourism development as a contributor to the economic development of Tasmania. National Environmental Research Programme, Landscapes and Policy Research, 161.

Javan, K. and Malazadeh, W. (2013). Surveying the tourism climate of Urmia City using bioclimatic 1ndicators, zagros quarterly. Journal of Urban and Urban Planning, 5, 16, 96-83. https://www.sid.ir/en/journal/ViewPaper.aspx ?ID=384905.

Jones, K.B, Riitters, K.H., Wickham, J.D., Tankersley, R.D., O’Neill, R.V., Chaloud, D.J., Smith, E.R., Neale, A.C. (1997). An ecological assessment of the United States Mid-Atlantic Region: a landscape atlas”. US Environmental Protection Agency. Report \# EPA 620/R-95/003.

Jurowski, C. (2010). Nature and ecotourism experience classification schema. ICHRIE Summer Conference Proceedings, August 28-31, San Juan, Puerto Rico. 
Kabataş, E. (2020). Determination of ecotourism potential in rural areas: Case of Kırklareli province Kofçaz district. Tekirdağ Namık Kemal University Graduate School of Natural and Applied Sciences Department of Landscape Architecture, Masters Thesis, Tekirdağ.

Khalid, M. D., Nasır, R. A. M., Ahmad, C. B. (2010). Recreation opportunity spectrum for ecotourism area At Rezab Hidupan Liar Fraser Hill Selangor. Research Management Institute (Rm1) Universiti Teknologi Mara, 43 p, Malezia.

Khalid, M., Hashim, Z. Rahman, A.A., Aziz, N.A. (2010). Ecotourism opportunity spectrum at sungai sedim recreational forest Area, Kedah. Center for Biodiversity and Sustainable, Universiti Teknologi Mara.

Kiper T. (2012). Advances in Landscape Architecture, In. Özyavuz, M. (Ed), Role of ecotourism in sustainable development. Rijeka, Crotia: IntechOpen Access Publisher.

Kiper T., Uzun O., Üstün Topal, T. (2017). Rural Development oriented ecotourism planning on catchment basin scale: the case of Pabuçdere and Kazandere catchment basins", Journal of Agricultural Science and Technology, 19, 293-305. http://jast.modares.ac.ir/article-23-454en.html

Kiper, T., Uzun, O., Özdemir, G., Üstün Topal, T. (2015). Ecotourısm plannıng for rural developmental purposes: the Kıyıöy Case. Tubitak Rapid Support Project No. 2130298 p.217. http://hdl.handle.net/123456789/3676.

Li, W. (2004). Environmental management indicators for ecotourism in China's nature reserves: a case study in Tianmushan nature reserve. Tourism Management, 25, 559-564. https://doi.org/10.1016/j.tourman.2003.06.001.

Mai, T. and Smith, C. (2015). Addressing the threats to tourism sustainability using systems thinking: a case study of Cat Ba Island, Vietnam. Journal of Sustainable Tourism, 23, 10, 1504-1528. https://doi.org/10.1080/09669582.2015.1045514 
McHarg, I. L. (1969). Design with Nature. Natural History Press, Garden City, New York.

Meydan, K. (2020). Determination of ecoturism potential with ecos method in Kastamonu provincial borders of Küre Mountains National Park. Kastamonu University Graduate School of Natural and Applied Sciences Department of Landscape Architecture Masters Thesis, Kastamonu.

Moeljad, A.S. (2015). Eco-tourism development strategy Baluran National Park in the Regency of Situbondo, East Java, Indonesia. IJERE, 4,4, 185-195.

Nahuelhual, L., Carmona, A., Lozada, P., Jaramilloc, A. and Aguayod, M. (2013). Mapping recreation and ecotourism as a cultural ecosystem service: An application at the local level in Southern Chile. Applied Geography, 40, 71-82. http://doi.org/10.1016/j.apgeog.2012.12.004.

Neth, B. (2008). Ecotourism as a tool for sustainable rural community development and natural resources management in the Tonle Sap Biosphere Reserve. Kassel: Kassel University Press. p. 277.

Ortiz-Guerrero, C. E. (2013). The new regionalism: policy 1mplications for rural regions. Cuadernos de Desarrollo Rural, 10, 70, 47-67.

Poyyamoli, G. (2018). Ecotourism policy in India: rhetoric and reality. Grassroots Journal of Natural Resources, 1,1, 46-61. http://dx.doi.org/10.33002/nr2581.6853.01015.

Rahman, A.A. (2014). Ecotourism opportunity spectrum (ECOS): a preliminary study on Lata Kijang Recreational Forest. Siri Kepelbagain Biologi Hutan Hutan Gunung Besar Hantu, Negeri Sembilan, Pengurusan Hutan, Persikitran Fizikal dan Kepelbagaian Biologi, Malaysia: Jabatan Perhutanan Semenanjung, pp.63-69

Ramazani Gourbi, B. (2010). The zonning of human bioclimatic comfort for ecotourism planning in Gilan, İran South Western of Caspian Sea. Australian Journal of Basic and Applied Sciences, 4,8, 3690-3694. https://www.sid.ir/en/Journal/ViewPaper.aspx ?ID=188571 
Roger, C. and Bhatta, K. (2013). Ecotourism planning and sustainable community development: theoretical perspectives for Nepal. SAJTH, 6,1, 69-96.

Rudianto, Putra, A.A., Akbar, Z. H., Putri, A. (2019). A strategic plan for developing the Banda Archipelago as an ecotourısm and environmental conservatıon areas. Jurnal Ilmu dan Teknologi Kelautan Tropis, 11, 3, 697-711. https://doi.org/10.29244/jitkt.v11i3.23861

Saaty, T. L. (2008). Decision making with the analytic hierarchy process. International Journal of Services Sciences, 1(1), 83-98. http://dx.doi.org/10.1504/IJSSCI.2008.017590

Safarabadı, A. (2016). Assessing ecotourism potential for sustainable development of coastal tourism In Qeshm Island, Iran. European Journal of Geography, 7, 4, 53 - 66.

Salıc1, A. (2018). Application of ecotourism opportunities spectrum method in ecotourism resources: a case study of Samandağ Coastal Areas in Southern Turkey. Applied Ecology and Environmental Research, 16(3), 2701 2715. http://dx.doi.org/10.15666/aeer/1603_27012715.

Sanır, F. (2000). Glossary of Geography Terms. Ankara: Gazi Publications.

Seifi, F. and Janbaz Ghobadi, G.R. (2017). The role of ecotourism potentials in ecological and environmental sustainable development of Miankaleh Protected Region. Open Journal of Geology, 7, 478-487. http://dx.doi.org/10.4236/ojg.2017.74033.

Suri, L. (2018). Natural threshold assessment criterı in plannıng. Istanbul Commerce University Journal of Science, 17(34), 47-67. https://dergipark.org.tr/tr/download/article-file/1195890.

Şahin, S. (2009). Human geography of Turkey. (Ed. S.Karabağ, S.Şahin), Human and Economic Geography of Turkey, Ankara: Gazi Publishing.

Tekeli, İ. (2016). As the urban-rural opposition disappears in the world and in Turkey, representation problems and strategy suggestions for settlements. Ankara: İdealkent Publishing.

Telfer, D. and Sharpley, R. (2008). Tourism and Development in the Developing World. London: Routledge. 
TIES, (2015). What is ecotourism - The International Ecotourism Society. https://ecotourism.org/what-is-ecotourism/.

Topay, M. (2003). A research on the evaluation of landscape features of Bartın-Uluyayla with respect to recreation-tourism uses. Ankara University Graduate School of Natural and Applied Sciences Department of Landscape Architecture Doctoral Thesis, Ankara.

Tran, L. and Walter, P. (2013). Ecotourism, gender and development in Northern Vietnam. Annals of Tourism Research. 44, 116-130. http://dx.doi.org/10.1016/j.annals.2013.09.005

Tümertekin, E. and Özgüç, N. (2015). Human geography: human culture space. İstanbul: Çantay Publishing.

Türker, N. (2013). Evaluatıon of Western Black Sea Regıon's ecotourısm resources and an ecotourısm route proposal. J. Acad. Soc. Sci. Stud. 6, 4, 1093- 1128.

Ullah, K. M. and Hafiz, R. (2014). Finding suitable locations for ecotourism development in Cox's Bazar using geographical information system and analytical hierarchy process. Geocarto International, 29, 3, 256-267. https://doi.org/10.1080/10106049.2012.760005.

Uzun, O., İlke, F., Çetinkaya, G., Erduran, F., Açıksöz, S. (2010). Konya, Bozkır Seydişehir-Ahırl1Yalıhüyük districts and Suğla Lake location Landscape Management, Conservation and Planning. TC Ministry of Forestry and Waterworks General Directorate of Nature Protection and National Parks, Laser Offset, p. 175.

Uzun, O., Müderrisoğlu, H., Demir, Z., Kaya, L.G., Gültekin, P., Gündüz, S. (2015). Yeşilırmak Basin landscape atlas project. Ministry of Forestry and Water Affairs, General Directorate of Nature Conservation and National Parks, Ankara. https://dx.doi.org/10.14744/planlama.2018.96967. 
Yenigül, S.B. (2017). New approaches in rural development policies and the effects of these approaches on Turkey's rural development policies. Planlama, 27, 1,16-25. http://doi.org/10.14744/planlama.2016.47450.

Yiğit, M. (2020). Evaluation of Giresun's ecotourism potential: proposal for an ecotourism route for sustainable tourism. Karabük University Department of Tourism Management Masters Thesis, Karabük.

Yüksel, A., Meral, A., Demir, Y., Eroğlu, E. (2020). Microcatchment Scale Landscape Evaluation in Capakcur Microcatchment. Turkısh Journal Of Agrıcultural and Natural Sciences, 7, 1, 16-26. https://doi.org/10.30910/turkjans.679893.

Zaman, M. (2010). Settlement geography. (Edit: C. Şahin) General Human and Economic Geography. Ankara: Gündüz Education and Publishing. 